\title{
An Invariant Based Approach to the Design of Hybrid Control Systems
}

\author{
Technical Report of the ISIS Group \\ at the University of Notre Dame \\ ISIS-2000-001 \\ February, 2000
}

\author{
James A. Stiver \\ Harris Corporation \\ Xenofon D. Koutsoukos \\ Department of \\ Melbourne, FL 32902 \\ Electrical Engineering \\ jstiver@harris.com \\ University of Notre Dame \\ Notre Dame, IN 46556 \\ xkoutsou@nd.edu
}

Panos J. Antsaklis

Department of

Electrical Engineering

University of Notre Dame

Notre Dame, IN 46556

antsaklis.1@nd.edu

Interdisciplinary Studies of Intelligent Systems 


\title{
An Invariant Based Approach to the Design of Hybrid Control Systems *
}

\author{
James A. Stiver \\ Harris Corporation \\ Melbourne, FL 32902, USA \\ jstiver@harris.com
}

\author{
Xenofon D. Koutsoukos \\ Department of Electrical Engineering \\ University of Notre Dame \\ Notre Dame, IN 46556, USA \\ xkoutsou@nd.edu
}

\author{
Panos J. Antsaklis \\ Department of Electrical Engineering \\ University of Notre Dame \\ Notre Dame, IN 46556, USA \\ antsaklis.1@nd.edu
}

\begin{abstract}
In this paper, a methodology for hybrid control design is presented. The hybrid systems of interest are characterized by a feedback architecture of a continuous nonlinear plant with a discrete-event controller. The natural invariants of the continuous dynamics are used to partition the state space into regions and to synthesize simple and efficient control laws. The paper contains a complete description of the approach including a description of the hybrid control system modeling, of the invariant based approach for the design of the partition, and of the controller synthesis methodology illustrated by examples. The implementation and optimization of the approach are also discussed. The approach presented in this paper not only describes a viable methodology to hybrid design, but also addresses fundamental issues in hybrid system theory that arise in reachability analysis and verification approaches that are based on partitions of the continuous state space. Finally, the developed techniques are adapted and applied to digital control systems.
\end{abstract}

\section{Introduction}

Hybrid systems are dynamical systems formed by interacting continuous and discrete dynamics and they arise from computer aided control of continuous processes in chemical and manufacturing, and also in areas such as computer and communication networks for example. They also arise from the hierarchical organization of complex control systems where the hierarchy requires less detailed models (discrete abstractions) of the functioning of the lower levels (continuous dynamics), necessitating the interaction of discrete and continuous components. Hybrid system analysis and controller synthesis techniques could provide an approach for design and verification of complex engineering systems and of intelligent control systems that exhibit highly autonomous behavior.

A convenient way to represent such hybrid control systems is shown in Figure 1. The continuous process to be controlled, together with any continuous controllers, are identified as the "Plant" and are typically described by differential/difference equations. The "Controller" is typically a discrete-event system described, for example by a finite automaton. The "Interface" makes it possible for these different processes to communicate with each other. This control framework is quite flexible and can describe modern engineering systems

*The partial financial support of the National Science Foundation (ECS95-31485) and the Army Research Office (DAAG5598-1-0199) is gratefully acknowledged. 


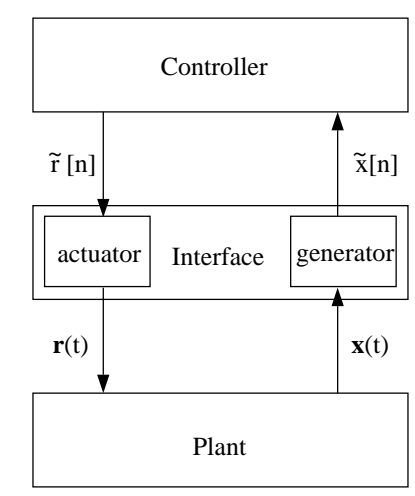

Figure 1: Hybrid Control System

where a computer process is used to control and coordinate several physical processes. It should be noted that the representation of Figure 1 is a functional representation that is convenient for the mathematical study of such hybrid systems. In certain systems, Figure 1 represents the actual control architecture and the controller, interface, and plant can be identified in a natural way. There are hybrid systems for which it may not be possible to separate the continuous from the discrete part in a natural way. In such systems, however, such separation is not so clear and in that case this representation is used primarily to study the system and identify its properties rather than to implement any control strategies.

IIt is generally assumed that the dynamic behavior of the plant is governed by a set of known nonlinear ordinary differential equations. In the model shown in Figure 1, the plant contains all continuous components of the hybrid control system, such as any conventional continuous controller that may have been developed, a clock if time and synchronous operations are to be modeled, and so on. The assumption that the plant contains only continuous components may seem restrictive since modern engineering system often contain parts that are used for advanced decision-making for example, and cannot be modeled effectively by differential/difference equations. It should be noted that the proposed approach does not intend to address problems that involve continuous controls, as it has been assumed that any continuous control action has already been considered and is included in the plant of Figure 1. It should also be noted that this approach does not address jumps in the continuous state that may occur when certain state variables are discontinuously reset. However, it includes switches where typically there are no jumps in the state value. There is however a large class of important engineering problems that can be solved using the approach described in this paper. The primary emphasis is on understanding how the interface between the continuous and the discrete part affects the properties of the hybrid system. This is one of the fundamental issues in the theory of hybrid control systems. These concepts and methodologies may be used directly or indirectly in the design of hybrid control systems, the latter in the case when the plant also contains discrete dynamics.

The interface plays a key role in determining the dynamic behavior of the hybrid control system. Here, the interface has been chosen to be simply a partitioning of the state space (see Figure 2) and this is done without loss of generality. If memory is necessary to derive an effective control law, it is included in the DES controller and not in the interface. Also the piecewise continuous command signal issued by the interface is simply a staircase signal as shown in Figure 3, not unlike the output of a zero-order hold in a digital control system. Note that signals such as ramps, sinusoids, etc., can be generated if desired by including an appropriate continuous system at (the input of) the plant. The simplicity of the interface with the resulting benefits in identifying central issues and concepts in hybrid control systems is perhaps the main characteristic of the approach.

In this paper, we present a methodology to design the partition of the continuous state space based on the natural invariants of the plant. Many times the partition of the state space is determined by physical constraints and it is fixed and given. Here, we assume that we can select the partition and we focus on specific problems for the interface design. Our approach leads to a systematic controller synthesis methodology that is illustrated in the paper using several examples. More details of the invariant based approach can be found in [22]. Earlier versions of this work have appeared in $[25,24,26]$. This paper contains a complete 


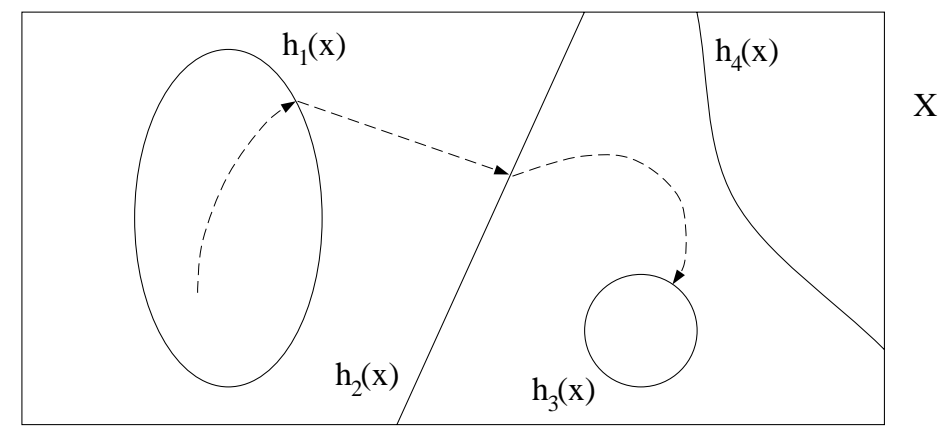

Figure 2: Partition of the continuous state space

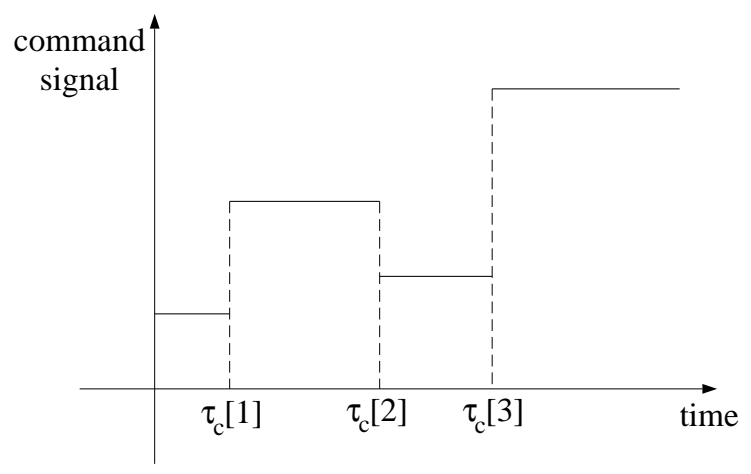

Figure 3: Command signal issued by the interface

and self-contained description of the approach including a computational procedure for implementation and illustrating examples. The approach presented here not only represents a viable methodology to hybrid design, but also addresses fundamental issues in hybrid system theory with respect to the partitioning of the continuous state space. Similar issues arise in reachability analysis and verification approaches that use continuous partitions based on the concept of the flow, see for example [1, 20, 28].

In general the design of the interface depends not only on the plant to be controlled, but also on the control policies available, as well as on the control goals to be attained. Certain control goals may require, for example, detailed feedback information while for others coarser quantization levels of the signals may be sufficient. The former case corresponds to finer partitioning of the feedback signal space, while the latter corresponds to coarser partitioning. The fact that different control goals may require different types of information about the plant is not surprising, as it is rather well known that to stabilize a system, for example, requires less detailed information about the system's dynamic behavior than to do tracking. Note that in general, the fewer the distinct regions in the partitioned signal space, the simpler (fewer states) the resulting DES plant model will be, and this will result in a simpler DES controller design. Since the systems to be controlled via hybrid controllers are typically complex, it is important to make every effort to use only the necessary information to attain the control goals, as this leads to simpler interfaces that issue only the necessary number of distinct symbols, and to simpler DES plant models and controllers. The question of systematically determining the minimum amount of information needed from the plant in order to achieve particular control goals using a finite number of distinct control policies is an important and largely open question; our work only partially resolves this question.

A great amount of research work has already been done in the hybrid systems area during the past decade; see for example $[4,2,5,3,7,6]$. A powerful approach that has been used extensively in the hybrid system literature uses the continuous partition (partition of the continuous state space) to suppress the continuous dynamics and to study the overall system in a discrete domain. A very important problem that arises is whether there exist partitions of a continuous system that capture the behavior in such a manner 
that preserve the important characteristics but result in simplified system descriptions. The design problem of continuous partitions is central in hybrid system and had been identified as such as early as 1991 [23]. The problem of obtaining discrete abstractions of continuous systems is directly related to the formal verification techniques for analyzing hybrid systems. In the computer science community, hybrid models are essentially extensions of finite state machines that incorporate simple continuous dynamics [1]. Verification algorithms are used to guarantee that the system model indeed satisfies the specifications. Since hybrid systems have infinite state spaces, the decidability of the verification algorithms is studied using discrete abstractions of hybrid systems. Verification algorithms for hybrid systems investigate how the flow of the continuous part interacts with certain regions in the state space. If it is assumed that the discrete variables are finite, then the decidability of the verification algorithms depends on whether each individual continuous component can be represented by a discrete abstraction preserving the behavior of interest. Several computational approaches have been proposed to determine finite bisimulations that are essentially equivalence relations on the state space and define continuous partitions $[11,16]$. Methods for computing continuous partitions based on approximations of the flow have also been considered [9]. Computational algorithms for the use of the phasespace geometric description of dynamics have been developed in [29]. Related approaches using a feedback architecture of a continuous plant with a discrete-event controller have appeared in $[21,27,10,17,8,15]$.

The paper is organized as follows. Section 2 presents the hybrid control system modeling. A methodology to design the controller and the interface together based on the natural invariants of a plant is described in Section 3. The invariant based approach for clocks is presented in Section 4. A methodology for optimizing the invariant based approach is discussed in Section 5. A computational procedure for the implementation of the approach is presented in Section 6. Finally, the case when the plant is described by a discrete-time system and the application of the approach to digital control is discussed in the Appendix.

\section{Hybrid Control System Modeling}

A hybrid control system, can be divided into three parts, the plant, interface, and controller as shown in Figure 1. In this model, the plant represents the continuous-time components of the system, while the controller represents the discrete-event portions. The interface is the necessary mechanism by which the former two communicate. The models used for each of these three parts, as well as the way they interact are now described.

\section{$2.1 \quad$ Plant}

The plant is the part of the model which represents the entire continuous portion of the hybrid control system. The distinguishing feature of the plant is that it has a continuous state space, where the states take on values that are real numbers, and evolve with time according to a set of differential equations. Motivated by tradition, this part of the model is referred to as the plant but since it contains all the continuous dynamics, it can also contain a conventional, i.e. continuous-time, controller.

The plant is in general a nonlinear, time-invariant system represented by a set of ordinary differential equations.

$$
\dot{\mathbf{x}}(t)=f(\mathbf{x}(t), \mathbf{r}(t))
$$

where $\mathbf{x}(t) \in \mathbf{X}$ and $\mathbf{r}(t) \in \mathbf{R}$ are the state and input vectors respectively, and $\mathbf{X} \subset \Re^{n}, \mathbf{R} \subset \Re^{m}$, with $t \in(a, b)$ some time interval. For each fixed $\mathbf{r}(t) \in \mathbf{R}$, the function $f(\cdot, r(t)): \mathbf{X} \rightarrow \mathbf{X}$ is continuous in $\mathbf{X}$ and meets the conditions for existence and uniqueness of solutions for initial states, $\mathbf{x}_{0} \in \mathbf{X}$. Note that the plant input and state are continuous-time vector valued signals. Boldface letters are used here to denote vectors and vector valued signals.

The representation of the plant is quite general and can be used to describe a large class of systems that includes time-invariant nonlinear systems and switching systems. For example, a linear switching system 
consisting of $m$ subsystems can be described by

$$
\dot{\mathbf{x}}(t)=\sum_{i=1}^{m} r_{i}(t) A_{i} \mathbf{x}(t)
$$

where $\mathbf{x} \in \mathbb{R}^{n}, r_{i}: \mathbb{R} \rightarrow\{0,1\}, \sum_{i=1}^{m} r_{i}(t)=1, \forall t \in \mathbb{R}$, where $r_{i}\left(\tau_{1}\right)=1$ implies that the system $A_{i}$ is actuated at time $\tau_{1}$.

\subsection{Controller}

The controller is a discrete event system which is modeled as a deterministic automaton [13]. This automaton is specified by a quintuple, $(\tilde{S}, \tilde{X}, \tilde{R}, \delta, \phi)$, where $\tilde{S}$ is the set of states, $\tilde{X}$ is the set of plant symbols, $\tilde{R}$ is the set of controller symbols, $\delta: \tilde{S} \times \tilde{X} \rightarrow \tilde{S}$ is the state transition function, and $\phi: \tilde{S} \rightarrow \tilde{R}$ is the output function. The symbols in set $\tilde{R}$ are called controller symbols because they are generated by the controller. Likewise, the symbols in set $\tilde{X}$ are called plant symbols and are generated based on events in the plant. The action of the controller is described by the equations

$$
\begin{aligned}
\tilde{s}[n] & =\delta(\tilde{s}[n-1], \tilde{x}[n]) \\
\tilde{r}[n] & =\phi(\tilde{s}[n])
\end{aligned}
$$

where $\tilde{s}[n] \in \tilde{S}, \tilde{x}[n] \in \tilde{X}$, and $\tilde{r}[n] \in \tilde{R}$. The index $n$ is analogous to a time index in that it specifies the order of the symbols in the sequence. The input and output signals associated with the controller are sequences of symbols. Tildes are used to indicate a symbol valued set or sequence. For example, $\tilde{X}$ is the set of plant symbols and $\tilde{x}[n]$ is the $n$th symbol of a sequence of plant symbols. Subscripts are also used, e.g. $\tilde{x}_{i}$ which denotes the $i$ th member of the symbol alphabet $\tilde{X}$.

\subsection{Interface}

The controller and plant cannot communicate directly in a hybrid control system because each utilizes a different type of signal. Thus an interface is required which can convert continuous-time signals to sequences of symbols and vice versa. The way that this conversion is accomplished determines, to a great extent, the nature of the overall hybrid control system. The interface consists of two simple subsystems, the generator and actuator.

The generator issues symbols to the controller and plays the role of a quantizer of the signals analogous to an A/D converter (sampler) in a digital control system. The actuator injects the appropriate control signal into the plant and it is analogous to a D/A converter (typically a zero-order hold) in a digital control system. The generator and the actuator perform, however, more general functions than their counterparts in a typical digital control system.

\subsubsection{Plant Events and the Generator}

The generator is the subsystem of the interface which converts the continuous-time output (state) of the plant to an asynchronous, symbolic input for the controller. To perform this task, two processes must be in place. First, a triggering mechanism is required which will determine when a plant symbol should be generated. Second, a process to determine which particular plant symbol should be generated is required.

In the generator, the triggering mechanism is based on the idea of plant events. A plant event is simply an occurrence in the plant, an idea borrowed from the field of discrete event systems. In the case of hybrid control, a plant event is defined by specifying a hypersurface which separates the plant's state space. The plant event occurs whenever the plant state trajectory crosses this hypersurface. The basis for this definition of a plant event is that an event is considered to be the realization of a specified condition. This condition can be given as an open region of the state space, separated from the remainder of the state space by a hypersurface. If the state crosses the hypersurface into the given open region, the event has 
occurred. Mathematically, the set of plant events recognized by the generator is determined by a set of smooth functionals, $\left\{h_{i}: \Re^{n} \rightarrow \Re, i \in I\right\}$, defined on the state space of the plant. Each functional must satisfy the condition,

$$
\nabla_{x} h_{i}(\xi) \neq 0, \forall \xi \in \mathcal{N}\left(h_{i}\right)
$$

which ensures that the null space of the functional, $\mathcal{N}\left(h_{i}\right)=\left\{\xi \in \Re^{n}: h_{i}(\xi)=0\right\}$, forms an $n-1$ dimensional smooth hypersurface separating the state space.

Let the sequence of plant events be denoted by $e$, where $e[n]=i$ means that the $n$th plant event was triggered by crossing the hypersurface defined by $h_{i}$. Let the sequence of plant event instants be given by $\tau_{e}$, where $\tau_{e}[n]$ is the time of the $n$th plant event and $\tau_{e}[0]=0$. By definition, these sequences satisfy the following conditions.

$$
e[n]=i \Rightarrow\left\{\begin{array}{l}
h_{i}\left(\mathbf{x}\left(\tau_{e}[n]\right)\right)=0 \\
\exists \delta_{1}>0 \text { s.t. } \forall \epsilon, 0<\epsilon<\delta_{1}, \pm h_{i}\left(\mathbf{x}\left(\tau_{e}[n]+\epsilon\right)\right)<0 \\
\exists \delta_{2}>0 \text { s.t. } \forall \epsilon, 0<\epsilon<\delta_{2}, \pm h_{i}\left(\mathbf{x}\left(\tau_{e}[n]-\delta_{2}\right)\right)>0, \pm h_{i}\left(\mathbf{x}\left(\tau_{e}[n]-\epsilon\right)\right) \geq 0
\end{array}\right.
$$

and

$$
\forall n, \tau_{e}[n]<\tau_{e}[n+1] \vee\left(\tau_{e}[n]=\tau_{e}[n+1] \wedge e[n]<e[n+1]\right)
$$

The first group, equation (5), contains three conditions: (i) at the time of the plant event the plant state lies on the triggering hypersurface, (ii) immediately after the event the plant state lies on the negative (positive) side of the triggering hypersurface, and (iii) prior to reaching the triggering hypersurface the plant state lied on the positive (negative) side. The fourth condition, equation (6) concerns the ordering of the sequences. It requires that plant events be ordered chronologically and simultaneous plant events be ordered according to their number, that is the value of $i$.

An alternative, and perhaps simpler, way of expressing the conditions of (5) is by the condition, $h_{i}(\mathbf{x}(t))=$ $0, \frac{d}{d t} h_{i}(\mathbf{x}(t)) \neq 0$. In this case the assumption is made that the derivative is nonzero, that is $\frac{d}{d t} h_{i}(\mathbf{x}(t)) \neq 0$ at the crossing. Note however that these conditions do not take into account the case where the crossing occurs exactly at an inflection point. When $\frac{d}{d t} h_{i}(\mathbf{x}(t))=0$, one must use (5).

A plant event will only cause a plant symbol to be generated if the hypersurface is crossed in the negative direction. The reason for this is that in many applications sensors only detect when a threshold is crossed in one direction, e.g. a thermostat. When the hypersurface is crossed in the opposite direction the event is silent, and for convenience, assume that a null symbol, $\epsilon$, is generated. At each time in the sequence $\tau_{e}[n]$, a plant symbol is generated according to the function $\alpha_{i}: \mathcal{N}\left(h_{i}\right) \rightarrow \tilde{X}$. The sequence of plant symbols can now be defined as

$$
\tilde{x}[n]=\left\{\begin{array}{cl}
\alpha_{i}\left(\mathbf{x}\left(\tau_{e}[n]\right)\right) & \text { nonsilent event } \\
\epsilon & \text { silent event }
\end{array}\right.
$$

where $i$ identifies the hypersurface which was crossed. Alternatively one could select the interface to generate information bearing symbols when crossed in either direction.

\subsubsection{The Actuator}

The actuator converts the sequence of controller symbols to a plant input signal, using the function $\gamma: \tilde{R} \rightarrow$ $\Re^{m}$, as follows.

$$
\mathbf{r}(t)=\sum_{n=0}^{\infty} \gamma(\tilde{r}[n]) I\left(t, \tau_{c}[n], \tau_{c}[n+1]\right)
$$

where $I\left(t, \tau_{1}, \tau_{2}\right)$ is a characteristic function taking on the value of unity over the time interval $\left[\tau_{1}, \tau_{2}\right)$ and zero elsewhere. $\tau_{c}[n]$ is the time of the $n$th control symbol which is based on the sequence of plant symbol instants, defined in equation (5), according to

$$
\tau_{c}[n]=\tau_{e}[n]+\tau_{d}
$$

where $\tau_{d}$ is the total delay associated with the interface and controller. Following the occurrence of a plant event, it takes a time of $\tau_{d}$ for a new control policy to be used by the plant. It will be assumed that $\tau_{e}[n]<\tau_{c}[n]<\tau_{e}[n+1]$. 
The plant input, $\mathbf{r}(t)$, can only take on certain constant values, where each value is associated with a particular controller symbol. Thus the plant input is a piecewise constant signal which may change only when a controller symbol occurs.

Remark In the interface a delay, $\tau_{d}$ was introduced. The presence of the delay is necessary for two reasons. First, from a practical point of view, the generator will not be able to detect an event until after the state has actually crossed the hypersurface. Second, if a chattering control strategy is used, the delay partially determines the chattering period. It is of course possible for two plant events to occur within the period of a single delay. In such a case each event will be acted upon, in turn, $\tau_{d}$ after it occurs. In this way the delay can pose a problem for the controller, but it is unavoidable as real systems cannot react instantaneously.

\section{Invariant Based Approach}

Here a methodology is presented to design the controller and the interface together based on the natural invariants of a plant described Equation (1). In particular, this section discusses the design of the generator, which is part of the interface, and the design of the controller. We assume that the plant is given, the set of available control policies is given, and the control goals are specified as follows. Each control goal for the system is given as a starting set and a target set, each of which is an open subset of the plant state space. To realize the goal, the controller must be able to drive the plant state from anywhere in the starting set to somewhere in the target set using the available control policies. Generally, a system will have multiple control goals. To successfully control the plant, the controller must know which control policy to apply and when to apply it. The controller receives all its information about the plant from the generator, and therefore the generator must be designed to provide that information which the controller requires.

We propose the following solution to this design problem. For a given target region, identify the states which can be driven to that region by the application of a single control policy. If the starting region is contained within this set of states, the control goal is achievable via a single control policy. If not, then this new set of states can be used as a target region and the process can be repeated. This will result in a set of states which can be driven to the original target region with no more than two control policies applied in sequence. This process can be repeated until the set of states, for which a sequence of control policies exists to drive them to the target region, includes the entire starting region (provided the set of control policies is adequate as mentioned below). When the regions have been identified, the generator is designed to tell the controller, via plant symbols, which region the plant state is currently in. The controller will then call for the control policy which drives the states in that region to the target region. Note that similar methods based based on backward analysis of the dynamics have been discussed in [20, 28].

\subsection{Generator Design}

To describe the regions mentioned above, we use the concept of the flow [19]. Let the flow for the plant (1) be given by $F_{k}: \mathbf{X} \times \Re \rightarrow \mathbf{X}$, where

$$
\mathbf{x}(t)=F_{k}(\mathbf{x}(0), t) .
$$

The flow represents the state of the plant after an elapsed time of $t$, with an initial state of $\mathbf{x}(0)$, and with a constant input of $\gamma\left(\tilde{r}_{k}\right)$. Since the plant is time invariant, there is no loss of generality when the initial state is defined at $t=0$. The flow is defined over both positive and negative values of time. The flow can be extended over time using the forward flow function, $F_{k}^{+}: \mathbf{X} \rightarrow \mathbb{P}\left(\mathbf{X}^{n}\right)$, and the backward flow function, $F_{k}^{-}: \mathbf{X} \rightarrow \mathbb{P}\left(\mathbf{X}^{n}\right)$, which are defined as follows.

$$
\begin{aligned}
& F_{k}^{+}(\boldsymbol{\xi})=\bigcup_{t \geq 0}\left\{F_{k}(\boldsymbol{\xi}, t)\right\} \\
& F_{k}^{-}(\boldsymbol{\xi})=\bigcup_{t \leq 0}\left\{F_{k}(\boldsymbol{\xi}, t)\right\}
\end{aligned}
$$


The backward and forward flow functions can be defined on an arbitrary set of states in the following natural way.

$$
\begin{aligned}
& F_{k}^{+}(\mathbf{A})=\bigcup_{\boldsymbol{\xi} \in \mathbf{A}}\left\{F_{k}^{+}(\boldsymbol{\xi})\right\} \\
& F_{k}^{-}(\mathbf{A})=\bigcup_{\boldsymbol{\xi} \in \mathbf{A}}\left\{F_{k}^{-}(\boldsymbol{\xi})\right\}
\end{aligned}
$$

where $\mathbf{A} \subset \mathbf{X}$. For a target region, $T, F_{k}^{-}(T)$ is the set of initial states from which the plant can be driven to $T$ with the input $\gamma\left(\tilde{r}_{k}\right)$. In addition, $F_{k}^{+}(T)$ is the set of states which can be reached with input $\gamma\left(\tilde{r}_{k}\right)$ and an initial state in $T$. Note that the backward and forward flow functions applied on set of states correspond to the precondition and postcondition operators used in verification algorithms of hybrid systems [1].

Now a generator design procedure can be described using the backward flow function. This is a preliminary procedure, upon which the final design method, developed subsequently, is based. For a given starting region, $S \subset \mathbf{X}$, and target region, $T \subset \mathbf{X}$, use the following algorithm.

1. If $S \subset T$, stop.

2. Identify the regions, $F_{k}^{-}(T), \forall \tilde{r}_{k} \in \tilde{R}$.

3. Let $T=\bigcup_{\tilde{r}_{k} \in \tilde{R}} F_{k}^{-}(T)$

4. Go to 1.

There are two problems associated with this algorithm as stated. First, it will not stop if there is no sequence of available control policies which will achieve the control goal, and second, actually identifying the regions given by the flow functions is quite involved. The first issue is related to the adequacy of the available control policies and will not be dealt with here. The second problem will be addressed. The difficulty in identifying a region given by a flow function is integrating over all the points in the target region. In the generator design procedure developed here, we will concentrate on finding a subset of the region $F_{k}^{-}(T)$, rather than the region itself. By definition, all the trajectories passing through $F_{k}^{-}(T)$ lead to the target region, $T$, and therefore all the trajectories found in a subset of $F_{k}^{-}(T)$ will also lead to the target. Here, we will focus on identifying subsets of $F_{k}^{-}(T)$ which we call common flow regions. Common flow regions are bounded by invariant manifolds and an exit boundary. The invariant manifolds are used because the state trajectory can neither enter nor leave the common flow region through an invariant manifold. The exit boundary is chosen as the only boundary through which state trajectories leave the common flow region.

To design the generator, it is necessary to select the set of hypersurfaces, $\left\{h_{i}: \mathbf{X} \rightarrow \Re \mid i \in I\right\}$ and the associated functions, $\left\{\alpha_{i}: \mathcal{N}\left(h_{i}\right) \rightarrow \tilde{R} \mid i \in I\right\}$, described in Section 2. These hypersurfaces make up the invariant manifolds and exit boundaries mentioned above, as well as forming the boundary for the target region(s). A target region, $T$, is specified as

$$
T=\left\{\boldsymbol{\xi} \in \mathbf{X}: \forall i \in I_{T}, h_{i}(\boldsymbol{\xi})<0\right\},
$$

where $I_{T}$ is the index set indicating which hypersurfaces bound the target region. A common flow region, $B$, is specified as

$$
B=\left\{\boldsymbol{\xi} \in \mathbf{X}: h_{i}(\boldsymbol{\xi})<0, h_{e}(\boldsymbol{\xi})>0, \forall i \in I_{B}\right\},
$$

where $I_{B}$ is an index set indicating which hypersurfaces form the invariant manifolds bounding $B$ and $h_{e}$ defines the exit boundary for $B$. The goal, of course, is that $B$ should include only states whose trajectories lead to the target region. Figure 4 shows an example of this where $I_{T}=\{1\}$ and $I_{B}=\{2,3\}$. The target region, $T$, is surrounded by $h_{1}$, the common flow region lies between $h_{2}$ and $h_{3}$ above the exit boundary, $h_{e}$.

We now present two propositions which can be used to determine the suitability of a set of hypersurfaces to achieve our goal of identifying a common flow region. In different situations, one of the propositions may be easier to apply than the other. The following propositions give sufficient conditions for the hypersurfaces bounding $B$ and $T$ to ensure that all state trajectories in $B$ will reach the target region. 


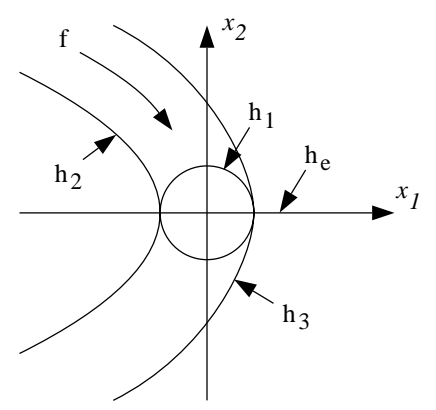

Figure 4: Target Region and Invariants

Proposition 1 Given the following:

1. A flow generated by a smooth vector field, $f_{k}$

2. A target region, $T \subset \mathbf{X}$

3. A set of smooth hypersurfaces, $h_{i}, i \in I_{B} \subset 2^{I}$

4. A smooth hypersurface (exit boundary), $h_{e}$

such that $B=\left\{\boldsymbol{\xi} \in \mathbf{X}: h_{i}(\boldsymbol{\xi})<0, h_{e}(\boldsymbol{\xi})>0, \forall i \in I_{B}\right\} \neq \emptyset$. For all $\boldsymbol{\xi} \in B$ there is a finite time, $t$, such that $F_{k}(\boldsymbol{\xi}, t) \in T$, if the following conditions are satisfied:

1. $\nabla_{\xi} h_{i}(\boldsymbol{\xi}) \cdot f(\boldsymbol{\xi})=0, \forall i \in I_{B}$

2. $\exists \epsilon>0, \nabla_{\xi} h_{e}(\boldsymbol{\xi}) \cdot f(\boldsymbol{\xi})<-\epsilon, \forall \boldsymbol{\xi} \in B$

3. $B \cap \mathcal{N}\left(h_{e}\right) \subset T$

Proof: The proof of this proposition is straightforward. The first condition of the proposition, which can be rewritten as

$$
\frac{d h_{i}(\mathbf{x}(t))}{d t}=0,
$$

precludes the state trajectory crossing any hypersurface indexed by the set $I_{B}$, thus ensuring no trajectory in $B$ will leave $B$ except through the remaining boundary. The second condition, which can be rewritten as

$$
\frac{d h_{e}(\mathbf{x}(t))}{d t}<-\epsilon
$$

ensures that within a finite time,

$$
t<\frac{h_{e}(\boldsymbol{\xi})}{\epsilon},
$$

the trajectory at $\boldsymbol{\xi} \in B$ will cross the exit boundary. The final condition guarantees that any trajectory leaving $B$ through the exit boundary will be in the target region when it does so. Together these conditions are sufficient to guarantee that any state in $B$ will enter the target region in finite time.

The second proposition uses a slightly different way of specifying a common flow region. In addition to the invariant manifolds and the exit boundary, there is also a cap boundary. The cap boundary is used to obtain a common flow region which is bounded. So for this case

$$
B=\left\{\boldsymbol{\xi} \in \mathbf{X}: h_{i}(\boldsymbol{\xi})<0, h_{e}(\boldsymbol{\xi})>0, h_{c}(\boldsymbol{\xi})<0, \forall i \in I_{B}\right\} .
$$


Proposition 2 Given the following:

1. A flow generated by a smooth vector field, $f_{k}$

2. A target region, $T \subset \mathbf{X}$

3. A set of smooth hypersurfaces, $h_{i}, i \in I_{B} \subset 2^{I}$

4. A smooth hypersurface (exit boundary), $h_{e}$

5. A smooth hypersurface (cap boundary), $h_{c}$

such that $B=\left\{\boldsymbol{\xi} \in \mathbf{X}: h_{i}(\boldsymbol{\xi})<0, h_{e}(\boldsymbol{\xi})>0, h_{c}(\boldsymbol{\xi})<0, \forall i \in I_{B}\right\} \neq \emptyset$ and $\bar{B}$ (closure of $B$ ) is compact. For all $\boldsymbol{\xi} \in B$ there is a finite time, $t$, such that $F_{k}(\boldsymbol{\xi}, t) \in T$, if the following conditions are satisfied:

1. $\nabla_{\xi} h_{i}(\boldsymbol{\xi}) \cdot f(\boldsymbol{\xi})=0, \forall i \in I_{B}$

2. $\nabla_{\xi} h_{c}(\boldsymbol{\xi}) \cdot f(\boldsymbol{\xi})<0, \forall \boldsymbol{\xi} \in B \cap \mathcal{N}\left(h_{c}\right)$

3. $B \cap \mathcal{N}\left(h_{e}\right) \subset T$

4. There are no limit sets in $\bar{B}$

Proof: As in Proposition 1, the first condition precludes the state trajectory crossing any hypersurface indexed by the set $I_{B}$, thus ensuring no trajectory in $B$ will leave $B$ except through one of the remaining boundaries. The second condition, which can be rewritten as

$$
\frac{d h_{c}(\mathbf{x}(t))}{d t}<0,
$$

ensures that no trajectory can leave $B$ through the cap boundary. Thus, the exit boundary provides the only available egress from $B$. The third condition guarantees that any trajectory leaving $B$ through the exit boundary will be in the target region when it does so. The final condition permits the application of a previously known result [18], stating that any state within a compact set without limit sets will leave that compact set in finite time.

Consider the hypersurfaces defined by $\left\{h_{i}: i \in I_{B}\right\}$. These hypersurfaces must first be invariant under the vector field of the given control policy, $f$. This can be achieved by choosing them to be integral manifolds of an $n-1$ dimensional distribution which is invariant under $f$. An $n-1$ dimensional distribution, $\Delta(\mathbf{x})$, is invariant under $f$ if it satisfies

$$
[f(\mathbf{x}), \Delta(\mathbf{x})] \subset \Delta(\mathbf{x}),
$$

where the $[f(\mathbf{x}), \Delta(\mathbf{x})]$ indicates the Lie bracket. Of the invariant distributions, those that have integral manifolds as we require, are exactly those which are involutive (according to Frobenius). This means

$$
\delta_{1}(\mathbf{x}), \delta_{2}(\mathbf{x}) \in \Delta(\mathbf{x}) \Rightarrow\left[\delta_{1}(\mathbf{x}), \delta_{2}(\mathbf{x})\right] \in \Delta(\mathbf{x}) .
$$

Therefore by identifying the involutive distributions which are invariant under the vector field, $f$, we have identified a set of candidate hypersurfaces. For details of these relationships between vector fields and invariant distributions, see [14]. Since an $n-1$ dimensional involutive distribution can be defined as the span of $n-1$ vector fields, over each of which it will then be invariant, and the control policy only gives one vector field, $f$, there will be more than one family of hypersurfaces which are all invariant under $f$. The set of all invariant hypersurfaces can be found in terms of $n-1$ functionally independent mappings which form the basis for the desired set of functionals, $\left\{h_{i}: i \in I_{B}\right\}$. This basis is obtained by solving the characteristic equation

$$
\frac{d x_{1}}{f_{1}(\mathbf{x})}=\frac{d x_{2}}{f_{2}(\mathbf{x})}=\cdots=\frac{d x_{n}}{f_{n}(\mathbf{x})}
$$

where $f_{i}(\mathbf{x})$ is the $i$ th element of $f(\mathbf{x})$. 


\subsection{Controller Design}

In previous work using this framework for hybrid control systems, the interface was assumed to be given and the controller was designed using the given plant and interface; see [27]. In those cases, the plant and interface were modeled as a discrete event system, called the DES plant model, and existing DES controller design techniques were adapted and used to obtain a controller. The drawback was that there was no guarantee that the desired behavior could be achieved with the given plant and interface. Now, with the generator design technique described in Section 3.1, the controller design is anticipated by the design of the interface. This represents an improvement over the previous situation because now there is no question that the control goal can be achieved once the interface has been successfully designed, and furthermore the actual controller design has been largely determined by the interface design.

Once the interface has been designed as described in Section 3.1, the design of the controller involves two steps. Here, it is assumed that adequate control policies are available. The first step is to construct one subautomaton for each control goal. This is the step which is already determined by the interface design. The second step is the connection of these subautomata to create a single DES controller. This step will depend upon the order in which the simpler control goals are to be achieved. For example, if a chemical process is to produce a sequence of different products, then each subautomaton in the controller would be designed to produce one of the products, and these subautomata would be connected to produce the products in the desired sequence. The hypersurfaces in the generator divide the state space of the plant into a number of cells. Two states are in the same cell exactly when they are both on the same side (positive or negative) with respect to each hypersurface. States which lie on a hypersurface are not in any cell.

The first step in creating the controller is the construction of the subautomata, one for each individual control goal. Each subautomaton is constructed in the following way.

i. Create a controller state to represent each cell.

ii. Place transitions between states which represent adjacent cells.

iii. Label each transition with the plant symbol which is generated by the hypersurface separating the associated cells.

We now have a subautomaton which can follow the progress of the plant state as it moves from cell to cell. Next the controller output function must be designed for each subautomaton. The controller symbol output by a given controller state depends on which common flow region contains the associated cell. Each common flow region was constructed using a specific control policy, and the control symbol which initiates that control policy should be output by controller states representing cells contained in that common flow region. However, in general, common flow regions will overlap, meaning a given cell can lie in more than one common flow region. In such cases treat the cell as lying within the common flow region which is closest to the target region. Distance, in this case, is the number additional control policies which must be used to reach the target region. If common flow regions are both the same distance, then the choice is arbitrary, though the common flow region which is favored in one case must then be favored in all such cases. States which represent cells not contained in any common flow region or target region will never be visited and can thus be deleted.

Once the individual subautomata have been constructed they must be connected to form a single controller. This can be accomplished by following these steps for each subautomaton.

i. Remove the state(s) which represent cells in the target region as well all transitions emanating from such states.

ii. Connect the dangling transitions to states in the subautomaton which achieves the next desired control goal. The connections will be to the states which represent the same cells as the states which were removed.

In this way, as soon as one control goal is achieved, the system will begin working on the next one. The actual order in which each control goals are pursued is up to the designer. 


\subsubsection{Example - Double Integrator}

Consider the double integrator example. Suppose we are given the plant,

$$
\dot{\mathbf{x}}(t)=\left[\begin{array}{ll}
0 & 1 \\
0 & 0
\end{array}\right] \mathbf{x}(t)+\left[\begin{array}{l}
0 \\
1
\end{array}\right] \mathbf{r}(t),
$$

three available control policies,

$$
\mathbf{r}(t) \in\{-1,0,1\},
$$

and the following control goal: drive the plant state to the interior of the unit circle from any initial point. So the starting set consists of the entire state space, and the target set is

$$
T=\left\{\boldsymbol{\xi} \in \mathbf{X}: \xi_{1}^{2}+\xi_{2}^{2}<1\right\} .
$$

The target set is bounded by the hypersurface given by

$$
h_{T}(\boldsymbol{\xi})=\xi_{1}^{2}+\xi_{2}^{2}-1
$$

The first step is to calculate the invariants which can be used to obtain hypersurfaces. There are three families of invariants, one for each of the three control policies.

$$
\begin{array}{r} 
\pm\left(\xi_{1}+\frac{1}{2} \xi_{2}^{2}+c_{1}\right) \\
\pm\left(\xi_{1}-\frac{1}{2} \xi_{2}^{2}+c_{2}\right) \\
\pm\left(\xi_{2}+c_{3}\right)
\end{array}
$$

The first hypersurface, $h_{1}$, is used to identify the target region.

$$
h_{1}(\boldsymbol{\xi})=h_{T}(\boldsymbol{\xi})=\xi_{1}^{2}+\xi_{2}^{2}-1
$$

A common flow region entering $T$ under the first control policy, $\mathbf{r}(t)=-1$, is bounded by

$$
\begin{array}{r}
h_{2}(\boldsymbol{\xi})=-\xi_{1}-\frac{1}{2} \xi_{2}^{2}-.9 \\
h_{3}(\boldsymbol{\xi})=\xi_{1}+\frac{1}{2} \xi_{2}^{2}-.9
\end{array}
$$

and

$$
h_{e}(\boldsymbol{\xi})=h_{4}(\boldsymbol{\xi})=\xi_{2}+.1
$$

These hypersurfaces satisfy Proposition 1. Identify this common flow region as $B_{1}$.

$$
B_{1}=\left\{\boldsymbol{\xi}: h_{i}(\boldsymbol{\xi})<0, h_{4}(\boldsymbol{\xi})>0, i \in\{2,3\}\right\}
$$

Likewise, a common flow region entering $T$ under the third control policy is bounded by

$$
\begin{array}{r}
h_{5}(\boldsymbol{\xi})=-\xi_{1}+\frac{1}{2} \xi_{2}^{2}-.9 \\
h_{6}(\boldsymbol{\xi})=\xi_{1}-\frac{1}{2} \xi_{2}^{2}-.9
\end{array}
$$

and

$$
h_{e}(\boldsymbol{\xi})=h_{7}(\boldsymbol{\xi})=-\xi_{2}+.1
$$

Identify this common flow region as $B_{2}$.

$$
B_{2}=\left\{\boldsymbol{\xi}: h_{i}(\boldsymbol{\xi})<0, h_{7}(\boldsymbol{\xi})>0, i \in\{5,6\}\right\}
$$




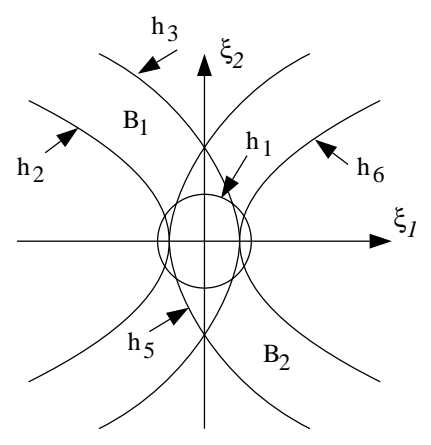

(i)

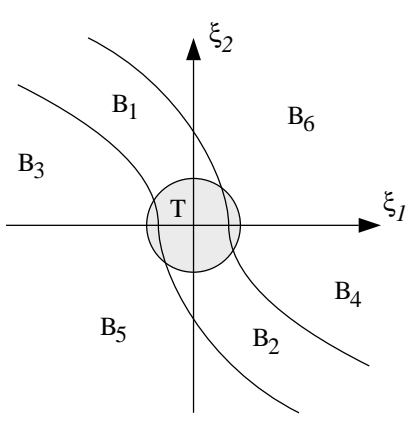

(ii)

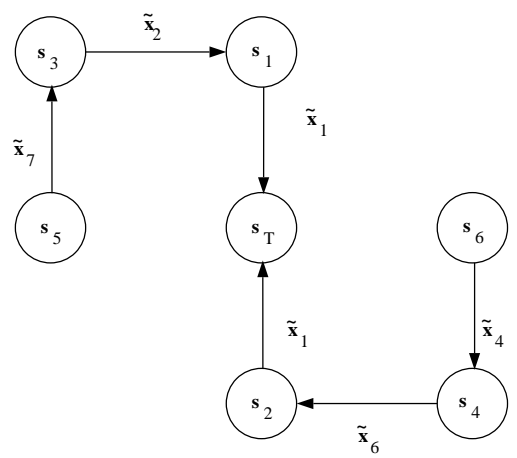

(iii)

Figure 5: (i) Target Region and Invariants, (ii) Final Regions for Double Integrator, (iii) Controller

Figure 5 (i) illustrates what we have so far. Now the target can be extended to include $B_{1}$ or $B_{2}$ and more common flow regions can be obtained. Let the new target be given by $T^{\prime}=T \cup B_{1}$. A common flow region entering $T^{\prime}$ under the second control policy is bounded by choosing

$$
I_{B_{3}}=\{7\}
$$

and $e=2$. A common flow region entering $T^{\prime \prime}=T^{\prime} \cup B_{3}$ under the third control policy is bounded by choosing

$$
I_{B_{5}}=\{6\}
$$

and $e=7$. Figure 5 (ii) gives a final picture of the hypersurfaces and regions involved in this example.

There is only one control goal for this example and therefore the entire controller will consist of a single subautomaton. Start by creating a controller state $\tilde{s}_{T}$ which is associated with the target region. Two common flow regions, labeled $B_{1}$ and $B_{2}$, were identified which lead to the target region. So create two more controller states, $\tilde{s}_{1}$ and $\tilde{s}_{2}$. $B_{1}$ consists of the trajectories which reach the target region under control policy $\tilde{r}_{1}$ and therefore $\phi\left(\tilde{s}_{1}\right)=\tilde{r}_{1}$, likewise $\phi\left(\tilde{s}_{2}\right)=\tilde{r}_{3}$. Connect $\tilde{s}_{1}$ to $\tilde{s}_{T}$ with a transition labeled $\tilde{x}_{1}$ which is generated when the plant state crosses $h_{1}$ to enter the target region. Do the same for $\tilde{s}_{2}$. Next, create $\tilde{s}_{3}$ to go with $B_{3}$, and add a transition to $\tilde{s}_{1}$ labeled $\tilde{x}_{2}$. When all the common flow regions have their associated states and transitions the controller shown in Figure 5 (iii).

\subsubsection{Example - Triple Integrator}

With the double integrator example, it is easy to see how the invariant surfaces are used. The technique can also be used in more complicated cases where it is not so intuitively obvious. Consider the triple integrator,

$$
\dot{\mathbf{x}}(t)=\left[\begin{array}{ccc}
0 & 1 & 0 \\
0 & 0 & 1 \\
0 & 0 & 0
\end{array}\right] \mathbf{x}(t)+\left[\begin{array}{l}
0 \\
0 \\
1
\end{array}\right] \mathbf{r}(t)
$$

with the same three available control policies,

$$
\mathbf{r}(t) \in\{-1,0,1\} .
$$

This time the control goal is to drive the state to the unit sphere from any initial state. First find a basis for the invariants by solving the characteristic equation

$$
\frac{d x_{1}}{\mathbf{x}_{2}}=\frac{d x_{2}}{\mathbf{x}_{3}}=\frac{d x_{3}}{\mathbf{r}}
$$




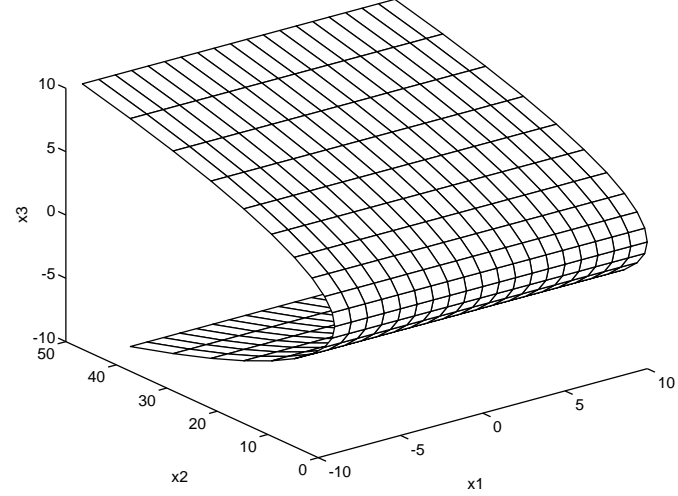

(i)

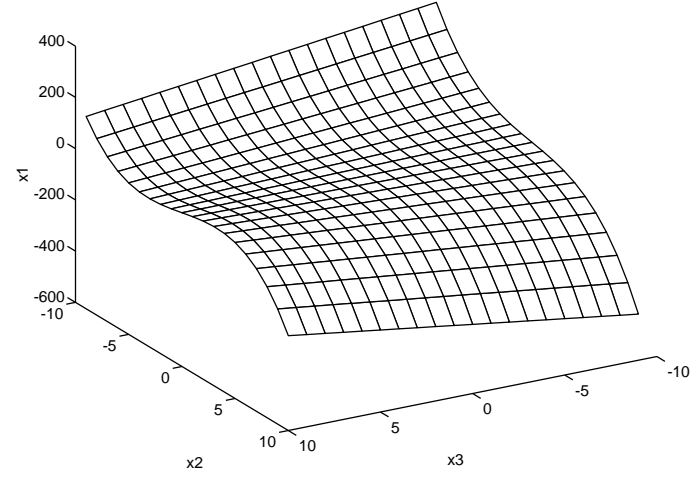

(ii)

Figure 6: (i) Invariant for $h_{a}$, (ii) Invariant for $h_{b}$

Two functions are obtained,

$$
\begin{array}{r}
h_{a}(\boldsymbol{\xi})=r \xi_{2}-\frac{1}{2} \xi_{3}^{2}+c_{1} \\
h_{b}(\boldsymbol{\xi})=r^{2} \xi_{1}+\frac{1}{3} \xi_{3}^{3}-r \xi_{2} \xi_{3}-r c_{2},
\end{array}
$$

where $r$ is the input (control policy) and $c_{1}$ and $c_{2}$ are arbitrary constants. Example hypersurfaces for $r=1$ and $c_{1}=c_{2}=0$ are shown in Figure 6 (i) and 6 (ii).

The target region is bounded by the hypersurface, $h_{1}$, i.e. $I_{T}=\{1\}$.

$$
h_{1}(\boldsymbol{\xi})=\xi_{1}^{2}+\xi_{2}^{2}+\xi_{3}^{2}-1
$$

Now identify a common flow region which enters the target region under the input $\mathbf{r}(t)=1$. The following hypersurfaces are used.

$$
\begin{aligned}
h_{2}(\boldsymbol{\xi}) & =-\xi_{2}+\frac{1}{2} \xi_{3}^{2}-\sqrt{2} \\
h_{3}(\boldsymbol{\xi}) & =\xi_{2}-\frac{1}{2} \xi_{3}^{2}-\sqrt{2} \\
h_{4}(\boldsymbol{\xi}) & =\xi_{1}+\frac{1}{3} \xi_{3}^{3}-\xi_{2} \xi_{3}-\sqrt{2} \\
h_{5}(\boldsymbol{\xi}) & =-\xi_{1}-\frac{1}{3} \xi_{3}^{3}+\xi_{2} \xi_{3}-\sqrt{2}
\end{aligned}
$$

The common flow region runs through the origin, where the target is centered, and the constant values, $\pm \sqrt{2}$, where chosen so that the common flow region passes through the target.

\section{The Invariant Based Approach for Clocks}

Recent attention in the area of hybrid systems has focused on a particular class of hybrid system referred to as linear hybrid systems or timed hybrid systems $[1,12]$. These systems all feature a continuous-time plant governed by

$$
\dot{\mathbf{x}}(t)=\mathbf{c}
$$

where $\mathbf{c} \in \Re^{n}$ is a constant vector and $\mathbf{c} \neq 0$. Here they will be referred to as integrators or clocks. 
Two important problems in the invariant based approach to interface design are now described. The first is the existence of the common flow regions. What is required is a set of conditions involving the target region and the control policy, under which a common flow region can be guaranteed to exist. Further conditions may be required to guarantee that a common flow region can be bounded by a set of invariant hypersurfaces. The second open problem is the construction of the hypersurfaces that bound the common flow region. Solutions to these problems for the general case have yet to be derived. The following section shows that these two problems are solved for the special case described above, where the plant is given by (53).

The advantage of using the plant characterized by equation (53) is that under a mild restriction (specified below), the common flow region (CFR) can be guaranteed to exist; furthermore the set of invariant hypersurfaces is readily determined. A systematic process exists to select a subset of these hypersurfaces to bound the common flow region. In the following, a particular approach is presented.

Notice that the following change of coordinates can be performed on the state space of the plant.

$$
\hat{\mathbf{x}}=P \mathbf{x}
$$

where

$$
P^{-1}=\left[\begin{array}{ccccc}
c_{1} & 0 & 0 & \cdots & 0 \\
c_{2} & 1 & 0 & \cdots & 0 \\
c_{3} & 0 & 1 & \ddots & \vdots \\
\vdots & \vdots & \ddots & \ddots & 0 \\
c_{n} & 0 & \cdots & 0 & 1
\end{array}\right]
$$

In the transformed coordinates, the plant is described by

$$
\dot{\hat{\mathbf{x}}}=P f\left(P^{-1} \hat{\mathbf{x}}\right)=\hat{f}(\hat{\mathbf{x}})=\left[\begin{array}{c}
1 \\
0 \\
\vdots \\
0
\end{array}\right] \text {. }
$$

More generally, the inverse of the transformation matrix can be $P^{-1}=[c, M]$, where $M$ is an $n$ by $n-1$ matrix such that $[c, M]$ is a nonsingular matrix.

In the transformed coordinates, the set of invariant hypersurfaces is given by the set of functions

$$
\{\hat{h}: \nabla \hat{h}(\hat{\mathbf{x}}) \cdot \hat{f}(\hat{\mathbf{x}})=0\}
$$

which can be simplified, due to the structure of $\hat{f}$, to

$$
\left\{\hat{h}: \frac{\partial \hat{h}(\hat{\mathbf{x}})}{\partial \hat{x}_{1}}=0\right\} .
$$

Therefore any $\hat{h}(\hat{\mathbf{x}})$ that is not a function of $\hat{x}_{1}$ will describe an invariant hypersurface in the transformed coordinates. The relationship between $\hat{h}$ and $h$ is defined by

$$
\hat{h}(\hat{\mathbf{x}}) \equiv h\left(P^{-1} \hat{\mathbf{x}}\right) .
$$

Assumption: Assume the target region is defined by a single invariant hypersurface, $h_{T}(\mathbf{x})$, in the original coordinates. A straightforward procedure then exists to select a single invariant hypersurface which bounds a common flow region for that target. This imposes only a mild loss of generality, because in most cases any desired region can be approximated in this way.

An outline of the procedure follows.

1. Use the coordinate transformation, $P$, to get the target region in the new coordinates according to equation (54). 
2. Select an exit boundary (hyperplane), normal to the $\hat{x}_{1}$ axis, which intersects the transformed target region. It will be of the form $\hat{h}_{e}(\hat{\mathbf{x}})=-\hat{x}_{1}+e$, for some $e \in \Re$.

3. Determine the curve that forms the intersection of the exit boundary and the target boundary. This curve is described by

$$
\left.\hat{h}_{T}(\hat{\mathbf{x}})\right|_{\hat{x}_{1}=e}=0, \hat{x}_{1}=e .
$$

4. Project the above curve onto the $\hat{x}_{1}=0$ hyperplane, along the $\hat{x}_{1}$ axis. This simply entails replacing the $\hat{x}_{1}=e$ with $\hat{x}_{1}=0$ in the expression for the curve.

5. The above projection, without the $\hat{x}_{1}=0$ condition,

$$
\left.\hat{h}_{T}(\hat{\mathbf{x}})\right|_{\hat{x}_{1}=e}=0,
$$

forms an invariant hypersurface bounding a common flow region in the transformed coordinates.

6. Apply the inverse transform of equation (54) to get the above hypersurface, as well as the exit boundary, in the original coordinates.

A modification of the above procedure can be used if the target region boundary consists of several hypersurfaces. In such a case, the target is considered to be the intersection of several regions, each of which is bounded by one of the hypersurfaces. The CFR will then consist of the intersection of the CFR's for each of the several regions. This means the final CFR will have a boundary consisting of several hypersurfaces just as the target region does.

Proposition 3 The hypersurface obtained in the above procedure identifies a common flow region for the target region, $T$.

Proof: First we prove that the first condition of Proposition 1 is satisfied. By construction the following relation holds in the transformed coordinate system.

$$
\nabla_{\hat{\mathbf{x}}} \hat{h}(\hat{\mathbf{x}}) \cdot \hat{f}(\hat{\mathbf{x}})=0
$$

Substituting for $\hat{h}$ and $\hat{f}$ reveals

$$
\nabla_{\hat{\mathbf{x}}} h\left(P^{-1} \hat{\mathbf{x}}\right) \cdot P f\left(P^{-1} \hat{\mathbf{x}}\right)=0 .
$$

Noting that

$$
\nabla_{\hat{\mathbf{x}}} h\left(P^{-1} \hat{\mathbf{x}}\right)=\nabla_{\mathbf{x}} h\left(P^{-1} \hat{\mathbf{x}}\right) P^{-1}
$$

this implies

$$
\nabla_{\mathbf{x}} h\left(P^{-1} \hat{\mathbf{x}}\right) P^{-1} \cdot \operatorname{Pf}\left(P^{-1} \hat{\mathbf{x}}\right)=0 .
$$

which can be simplified to yield the first condition of Proposition 1

$$
\nabla_{\mathbf{x}} h(\mathbf{x}) \cdot f(\mathbf{x})=0 .
$$

Second, we prove that the second condition of Proposition 1 is satisfied. Since we have defined $\hat{f}(\hat{\mathbf{x}})$ according to $(56)$, and chosen $\hat{h}_{e}(\hat{\mathbf{x}})=-\hat{x}_{1}+e$, we know that

$$
\nabla_{\hat{\mathbf{x}}} \hat{h}_{e}(\hat{\mathbf{x}}) \cdot \hat{f}(\hat{\mathbf{x}})=-1
$$

By the same steps as followed in the first part of this proof, we therefore know that

$$
\nabla_{\mathbf{x}} h_{e}(\mathbf{x}) \cdot f(\mathbf{x})=-1
$$

which satisfies condition two of Proposition 1 for $\epsilon<1$. Finally, the third condition of the proposition is satisfied because the invariant hypersurface is chosen, based on the intersection of the target region and the exit boundary, to satisfy it. 
The only portion of the above procedure which requires some heuristic effort is step 2. The selection of the exit boundary will determine the cross section of the common flow region. This is because the CFR boundary will intersect the target region boundary along the same curve that the exit boundary intersects the target region boundary. Next, another procedure is presented, which requires no heuristic effort and is optimal in the sense that is finds the largest possible common flow region that exists. This procedure is more difficult to implement.

1. Use the coordinate transformation, $P$, to get the target region in the new coordinates according to equation (54).

2. Select an exit boundary (hyperplane), normal to the $\hat{x}_{1}$ axis, which intersects the transformed target region. It will be of the form $\hat{h}_{e}(\hat{\mathbf{x}})=-\hat{x}_{1}+e$, where $e \in \Re$.

3. Project the target region boundary onto the $\hat{x}_{1}=0$ hyperplane, along the $\hat{x}_{1}$ axis.

4. Determine the curve on the $\hat{x}_{1}=0$ hyperplane that forms the boundary of the above projected region.

5. The above curve, without the $\hat{x}_{1}=0$ condition, forms an invariant hypersurface bounding a common flow region in the transformed coordinates.

6. Apply the inverse transform of equation (54) to get the above hypersurface, as well as the exit boundary, in the original coordinates.

\subsection{Example}

Given the following plant

$$
\dot{\mathbf{x}}=\left[\begin{array}{l}
c_{1} \\
c_{2} \\
c_{3}
\end{array}\right]
$$

with $c_{1} \neq 0$, let the transformation matrix be

$$
T=\left[\begin{array}{ccc}
\frac{1}{c_{1}} & 0 & 0 \\
-\frac{c_{2}}{c_{1}} & 1 & 0 \\
-\frac{c_{3}}{c_{1}} & 0 & 1
\end{array}\right]
$$

Let the target region be defined by the unit sphere centered at the origin. It is bounded by the hypersurface

$$
h_{T}(\mathbf{x})=x_{1}^{2}+x_{2}^{2}+x_{3}^{2}-1 .
$$

In transformed coordinates it becomes

$$
\begin{aligned}
\hat{h}_{T}(\hat{\mathbf{x}}) & =\left(c_{1} \hat{x}_{1}\right)^{2}+\left(c_{2} \hat{x}_{1}+\hat{x}_{2}\right)^{2}+\left(c_{3} \hat{x}_{1}+\hat{x}_{3}\right)^{2} \\
& =\left(c_{1}^{2}+c_{2}^{2}+c_{3}^{2}\right) \hat{x}_{1}^{2}+\hat{x}_{2}^{2}+\hat{x}_{3}^{2}+2 c_{2} \hat{x}_{1} \hat{x}_{2}+2 c_{3} \hat{x}_{1} \hat{x}_{3}-1
\end{aligned}
$$

The exit boundary is a plane normal to the $\hat{x}_{1}$ axis that intersects the target region. For simplicity, the exit boundary is chosen as the $\hat{x}_{1}=0$ hyperplane, which is convenient because the projection is then trivial. Projection onto the $\hat{x}_{1}=0$ hyperplane yields the following curve

$$
\hat{x}_{2}^{2}+\hat{x}_{3}^{2}-1=0, \hat{x}_{1}=0 .
$$

The common flow region is bounded by

$$
\hat{h}_{B}(\hat{\mathbf{x}})=\hat{x}_{2}^{2}+\hat{x}_{3}^{2}-1 .
$$

Finally, the inverse transform is used to obtain the hypersurfaces in the original coordinates.

$$
\begin{aligned}
h_{B}(\mathbf{x}) & =\left(x_{2}-\frac{c_{2}}{c_{1}} x_{1}\right)^{2}+\left(x_{3}-\frac{c_{3}}{c_{1}} x_{3}\right)^{2}-1 \\
h_{e}(\mathbf{x}) & =\frac{1}{c_{1}} x_{1}
\end{aligned}
$$

Figure (7) shows the target regions and CFR's in both the original and transformed coordinates. 


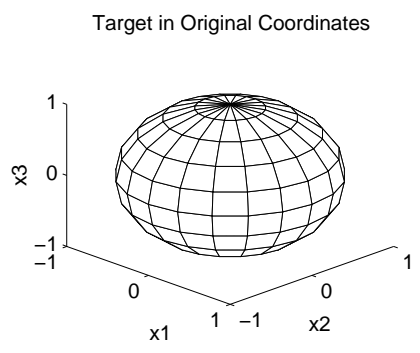

CFR in Transformed Coordinates

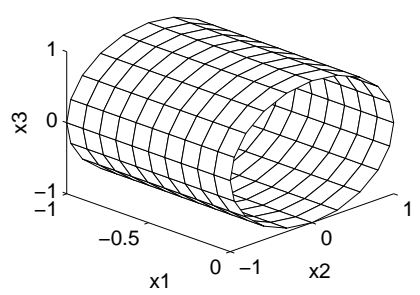

Target in Transformed Coordinates

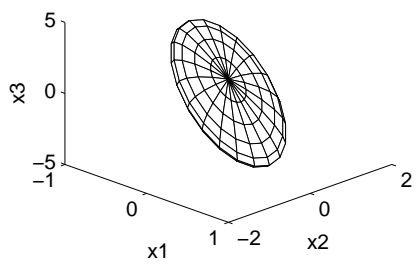

CFR in Original Coordinates

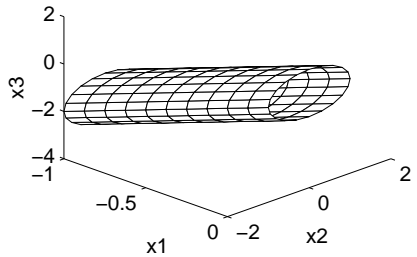

Figure 7: Regions for the Example

\section{Optimizing the Invariant Based Approach}

The invariant based approach to interface design produces a set of hypersurfaces in the state space of the plant. These hypersufaces form the boundaries of common flow regions (CFRs), which are regions of the state space where all the trajectories flow to the same subsequent CFR under a particular control policy. By driving the state through a sequence of CFRs the controller can eventually drive it to the target region. Since each CFR is associated with a unique control policy, the controller has only determine the CFR in which the current state resides and apply the associated policy.

In general, however, the CFRs will intersect each other, and therefore the plant state may well lie in multiple CFRs simultaneously. In such cases the controller must choose one of the CFRs in order to decide which control policy to apply. The controller design procedure described here bases this choice on the optimization of some measure of performance. The particulars of the measure used are not important to the design procedure provided they can be expressed as a cost associated with each CFR. The controller will choose to drive the plant through the sequence of CFRs which reaches the target region with the lowest total cost.

\subsection{Problem Formulation}

Assume the invariant based design scheme has been completed. The result is a set of hypersurfaces which identify a set of CFRs. The set of CFRs is denoted by $\mathcal{T}$ where $\mathcal{T} \subset \mathbb{P}(\mathbf{X})$. For each CFR, $T \in \mathcal{T}$, three functions are defined.

$$
\begin{array}{cl}
\operatorname{depth}(\mathrm{T}) \in\{0 . . D\} & \text { depth of CFR T } \\
\operatorname{cost}(\mathrm{T}) \in \Re & \text { cost of traversing } \mathrm{T} \\
C P(\mathrm{t}) \in\{0 . . K\} & \text { control policy for } \mathrm{T} \\
\operatorname{next}(\mathrm{T}) \in \mathcal{T} & \text { CFR reached via CFR } \mathrm{T}
\end{array}
$$

These functions are determined by the interface design algorithm. The depth of a CFR refers to the number of CFRs traversed before reaching the target. The target has a depth of 0 . The cost of traversing a CFR will depend on the particular control problem, it can reflect the control effort required, the performance of the plant when its state lies in the CFR, etc. The function, next, indicates which CFR is reached from the present CFR. This function can be extended to in the following way. 


$$
\begin{aligned}
& \operatorname{next}^{0}(\mathrm{~T})=\mathrm{T} \\
& \operatorname{next}^{1}(\mathrm{~T})=n \operatorname{ext}(\mathrm{T}) \\
& \operatorname{next}^{2}(\mathrm{~T})=\operatorname{next}(\operatorname{next}(\mathrm{T}))
\end{aligned}
$$

The set of hypersurfaces which form the boundaries for the various CFRs will form a set of open regions, $\mathcal{S} \subset \mathbf{P}(\mathbf{X})$, in the plant state space. Each of these open regions may belong to a number of CFRs. Through observation of the sequence of plant events the controller knows which of these open regions contains the plant state an must decide which control policy to apply. The following function identifies the CFRs which contain a given open region, $S$.

\section{$C F R(\mathrm{~S}) \subset \mathcal{T} \quad$ CFRs containing region $\mathrm{S}$}

The relation $T \in C F R(\mathrm{~S})$ is equivalent to $S \in T$.

\subsection{Optimization}

When the plant state is in the open region, $S$, the controller will select from among the CFRs in the set $C F R(\mathrm{~S})$, the CFR which provides the lowest total cost to reach the target. The total cost to reach the target from CFR $\mathrm{T}$ is given by the following summation.

$$
\sum_{i=0}^{\operatorname{depth}(T)} \operatorname{cost}\left(n \operatorname{ext} t^{i}(T)\right)
$$

Therefore when the plant state is in the open region $\mathrm{S}$, the controller will apply the control policy

$$
C P(T)
$$

where $\mathrm{T}$ is determined by the following optimization,

$$
\min _{T \in C F R(S)} \sum_{i=0}^{\operatorname{depth}(T)} \operatorname{cost}\left(n \operatorname{ext} t^{i}(T)\right) .
$$

\subsection{Designing the Optimal Controller}

The most straightforward way to implement the controller is to create an automaton with one state for each of the open regions in the set, $\mathcal{S}$. Then as the plant evolves and produces plant symbols, the controller can track the plant state and provide the optimal control policy as defined by (58). Therefore the output function for the controller is given by

$$
\phi(S)=C P(T)
$$

where $\mathrm{T}$ is given by (58).

Remark: Using this controller design scheme, it is possible for the controller to change its mind after selecting a CFR as optimal. If, while traversing the selected CFR, the plant state passes through another CFR the controller may find a lower cost in the new CFR and abandon the previous one.

\section{A Computational Procedure for the Invariant Based Approach}

The design procedure presented in this paper is straightforward, but not computationally easy. For these reasons it is desirable to automate the procedure on a computer. Here a simple automation scheme is presented along with results for an example of an unmanned underwater vehicle (UUV). The UUV demonstrates that the algorithm also works for more complex systems where implementation via human calculation is all but impossible. The procedure was developed in [22] and is similar to the backward analysis approach in formal verification [1]. 


\subsection{The Algorithm}

The computerized procedure does not find the hypersurfaces which bound the common flow regions, but rather seeks to identify the regions directly. This is achieved by simply back-calculating the state trajectories from the target region and recording the states which are encountered. It requires quantizing and bounding the state space so that the computer will have a finite number of state values to deal with.

To use the program the designer must choose the quantization levels for each state, $\Delta x_{i}$, and the range of each state, $x_{i, \min }$ and $x_{i, \max }$. These choices lead to a quantization of the plant state space into a number of $\mathrm{n}$-dimensional cells. The number of cells is given by

$$
\prod_{i} \frac{x_{i, \max }-x_{i, \min }}{\Delta x_{i}}
$$

As can be seen, the order of the computational complexity is $q^{n}$, where $q$ is the number of quantization levels and $n$ is the number of states. This limits the size of systems which can be handled in a reasonable amount of time. The limit depends on the computational power available and the on the designer's idea of what is "reasonable". For example, for the UUV system we have $q^{n} \approx 10^{5}$.

After the designer has quantized and bounded the state space, the procedure requires two additional pieces of information from the designer. The set of available plant inputs (control policies) must be provided and the target region must be specified in terms of the cells. That is, the cells which lie in the target region must be identified as such by the designer. Once the procedure has the requisite information, it proceeds according to the algorithm outlined by the flowchart shown in Figure 8. The algorithm shown in the chart will locate all cells from which it is possible to reach the target region via the application of any one control policy. The procedure resets the target region to include all these cells and then repeats the algorithm. In this way, all cells from which it is possible to reach the original target via the application of two control policies in sequence are identified. The algorithm will be repeated as many times as the designer has specified. When it terminates, each cell is marked with the control policy which should be used to reach the target, either directly or as the first in a sequence of control policies.

There is generally more than one trajectory which can be followed from an initial point to a target region. A question therefore arises regarding which of the possible trajectories should be followed. A trajectory that satisfies a desirable cost criterion can be obtained using the optimization procedure presented in Section 5 . An optimization procedure can be formulated by defining a cost function to associate costs with trajectories. Then, when the algorithm calculates a trajectory it will also calculate the associated cost and compare it to any trajectories which have been found previously. The trajectory with the lowest cost would be retained. In the UUV example, the chosen trajectory was simply the first one which was found by the algorithm.

The procedure has a requirement that the search area of the state space be bounded, and this requirement can be used to an advantage by the designer. First, the speed of the procedure is increased by shrinking the size of the state space which is searched. In addition, by carefully choosing the shape of the search area, the designer can coerce the procedure into finding control laws which satisfy a desired property. For example, in a forbidden region problem, the undesirable region is placed outside the bounds of the search area and therefore no trajectories in this region will be considered. This idea can be extended to force the procedure to find a solution which follows a rough path from the starting set to the target set. The designer can accomplish this by defining a "tube" through the state space from the starting set to the target set, and using the boundary of the tube as the boundary of the search area in the state space. Of course, reducing the search area reduces the number of possible solutions and it is possible that no solutions lie in a particular region of the state space.

\subsection{Example - Unmanned Underwater Vehicle}

Unmanned underwater vehicles (UUV) have a wide variety of practical usages in missions where it is dangerous or impossible to send a manned underwater vehicle. Exploration, search and rescue, salvage, mine disposal, and demolition are some examples of the uses of UUV's. The UUV shows that the program described above can also solve a reasonably complex, nonlinear system. 


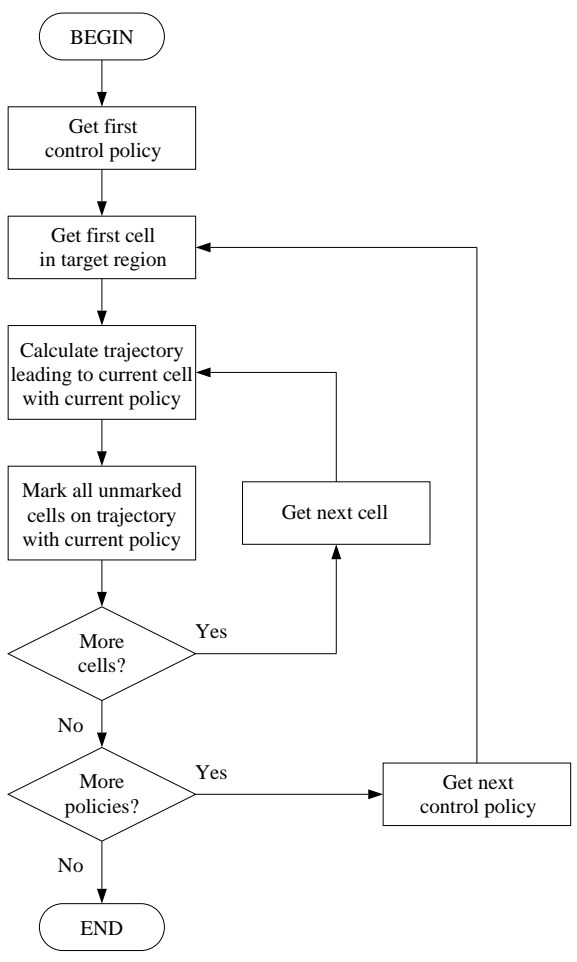

Figure 8: Algorithm

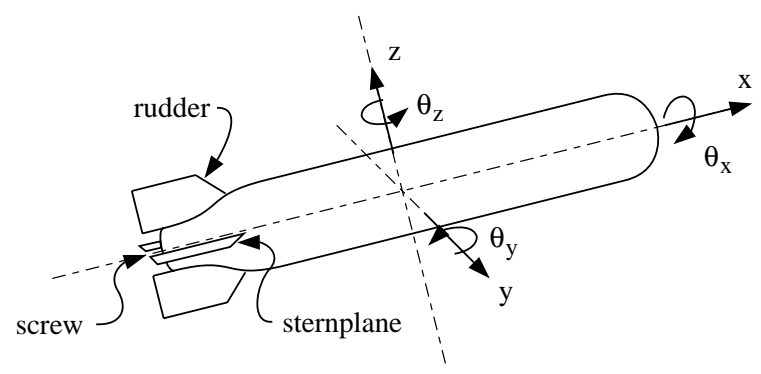

Figure 9: Unmanned Underwater Vehicle

This example used a simplified model of a six-degree-of-freedom UUV depicted in Figure 9. The three types of linear displacement are surge, sway, and heave, which represent translation in the $\mathrm{x}, \mathrm{y}$, and $\mathrm{z}$, directions respectively. The three types of angular displacement are roll, pitch, and yaw. The model employed here has six states which are the time derivatives of the three linear displacements and the magnitudes of the three angular displacements. By expanding to a nine state model, the magnitudes of the linear displacements, could also be included. They are omitted here to simplify the control problem and because they do not affect the dynamics of the UUV.

The UUV model has three inputs which control the rudder, stern plane, and screw. The following table summarizes the variables of the model. 


$\begin{array}{ll}x & \text { surge rate (forward speed) } \\ y & \text { sway rate (lateral speed) } \\ z & \text { heave rate (vertical speed) } \\ \theta_{x} & \text { roll angle in radians } \\ \theta_{y} & \text { pitch angle in radians } \\ \theta_{z} & \text { yaw angle in radians } \\ u_{x} & \text { screw } \\ u_{y} & \text { stern plane angle } \\ u_{z} & \text { rudder angle }\end{array}$

The simplified model is as follows.

$$
\begin{aligned}
\dot{x} & =-x+u_{x} \\
\dot{y} & =-y+0.01 x u_{y} \\
\dot{z} & =-z+0.01 x u_{z} \\
\dot{\theta}_{y} & =0.15 x u_{y} \\
\dot{\theta}_{z} & =0.15 x u_{z}
\end{aligned}
$$

Notice that the roll angle is not included in the model. It is assumed that the center of mass of the UUV is sufficiently far beneath the center of buoyancy so that the roll angle is always zero.

To control the UUV, the actuator can implement ten different control policies. The policies allow various combinations of two screw speeds (on and off), three stern plane positions (up, level, down), and three rudder positions (left, right, straight).

$$
\mathbf{r}(t)=\left[\begin{array}{l}
u_{x} \\
u_{y} \\
u_{z}
\end{array}\right]
$$

where $u_{x}=1, u_{y} \in\{-1,0,1\}, u_{z} \in\{-1,0,1\}$, or $u_{x}=u_{y}=u_{z}=0$. The unmanned underwater vehicle example is sufficiently complex, with five states, three inputs, and non-linear dynamics, that the design methodology of this paper cannot be carried out without some form of systematic, automated procedure.

The procedure is used to design a controller for the UUV and then the design is evaluated through simulation. The state space of the UUV plant is quantized and bounded as follows.

$$
\begin{aligned}
& x_{1, \min }=0 \quad x_{1, \max }=1 \quad \Delta x_{1}=0.1 \\
& x_{2, \min }=-0.5 \quad x_{2, \max }=0.5 \quad \Delta x_{2}=0.2 \\
& x_{3, \min }=-0.5 \quad x_{3, \max }=0.5 \quad \Delta x_{3}=0.2 \\
& x_{4, \min }=-\pi / 2 \quad x_{4, \max }=\pi / 2 \quad \Delta x_{4}=\pi / 20 \\
& x_{5, \min }=-\pi / 2 \quad x_{5, \max }=\pi / 2 \quad \Delta x_{5}=\pi / 20
\end{aligned}
$$

The controller has ten control policies to choose from. These policies are obtained by combining two propeller speeds $u_{x} \in\{0,1\}$, three stern plane angles $u_{y} \in\{-10,0,10\}$, and three rudder angles $u_{z} \in$ $\{-10,0,10\}$. Of the control policies with $r_{1}=0$, only the one with $r_{2}=r_{3}=0$ is kept, reducing the total number of control policies from eighteen to ten.

The target region consists of the following interval.

$$
\left[\begin{array}{c}
0.1 \\
-0.1 \\
-0.1 \\
-\pi / 40 \\
-\pi / 40
\end{array}\right]<\mathbf{x}<\left[\begin{array}{c}
0.2 \\
0.1 \\
0.1 \\
\pi / 40 \\
\pi / 40
\end{array}\right]
$$



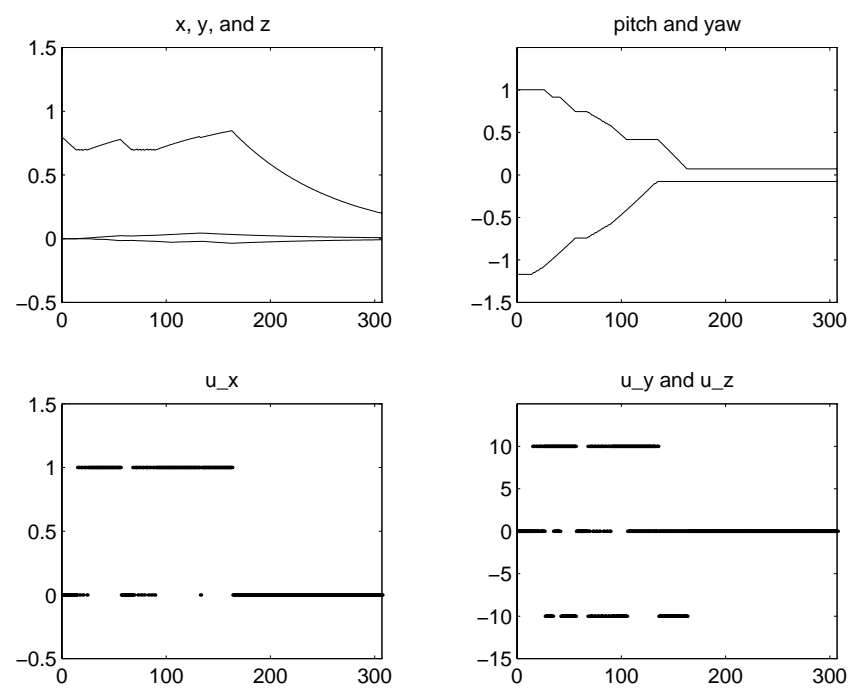

Figure 10: UUV Simulation \#1

The results of two simulations are presented here. The two trials have the following initial conditions.

$$
\mathbf{x}=\left[\begin{array}{c}
\text { surge } \\
\text { sway } \\
\text { heave } \\
\text { pitch } \\
\text { yaw }
\end{array}\right], \mathbf{x}_{1}=\left[\begin{array}{c}
0.8 \\
0 \\
0 \\
-1.17 \\
1.00
\end{array}\right], \mathbf{x}_{2}=\left[\begin{array}{c}
0.2 \\
0.4 \\
0 \\
0.67 \\
-1.50
\end{array}\right]
$$

Results of two trials are shown in Figure 10 (i) and 11. Each figure consists of four graphs. The graph in the upper left shows the surge, sway, and heave over time. The graph in the upper right shows the pitch and yaw over time. The trajectories on these two graphs can be distinguished by noting the initial conditions. The two lower graphs show the control signal. On the lower left is the propeller speed which is either 0 or 1. The segments which appear to overlap reveal the presence of chattering (due to quantization). The final graph shows the stern plane angle and the rudder angle. In Figure 10, the stern plane switches between 0 and 10 and the rudder angle switches between -10 and 0 . In Figure 11 these are reversed. As can be seen, the basic control strategy which developed is simply to accelerate and turn until the pitch and yaw are within the bounds of the target region, and then coast until the forward speed is also in the target.

\section{Conclusions}

An invariant based methodology for hybrid control design is presented. The hybrid systems of interest are characterized by a feedback architecture of a continuous nonlinear plant with a discrete-event controller. The natural invariants of the continuous dynamics are used to partition the state space into regions and to synthesize simple and efficient control laws. The primary emphasis is on understanding how the interface between the continuous and the discrete part affects the properties of the hybrid system. This is one of the fundamental issues in the theory of hybrid control systems. This paper contains a complete and selfcontained description of the approach for the case when a discrete-event controller is used to supervise a continuous plant. The fundamental issues studied here are important for the design of complex engineering applications even when the plant contain discrete dynamics. 

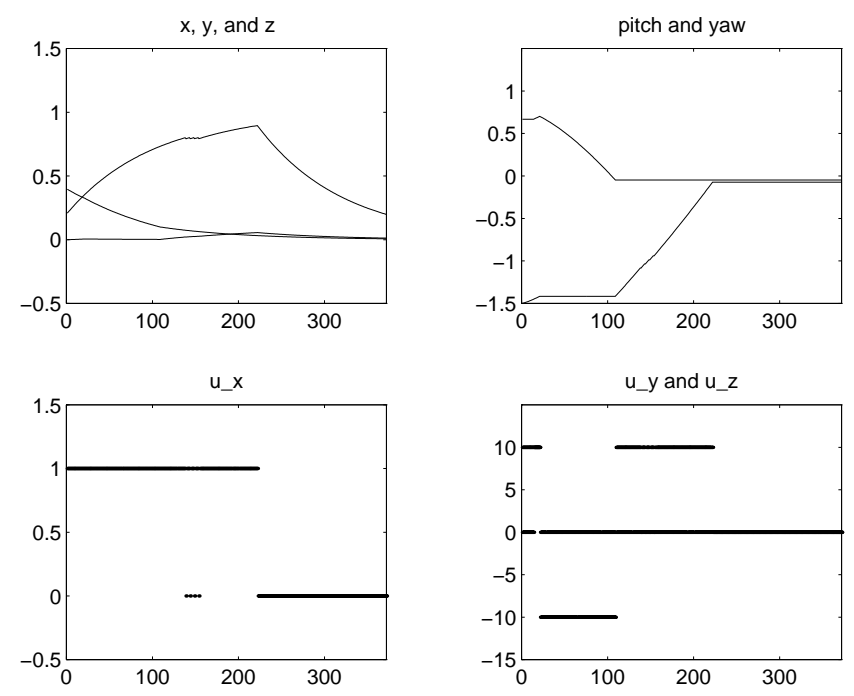

Figure 11: UUV Simulation \#2

\section{A Appendix}

\section{A.1 Modeling with Discrete-Time Plant}

In Section 2 the modeling framework for hybrid control systems contained a continuous-time plant. It is also possible to model hybrid control systems with discrete time plants. This appendix presents a model development, where the plant is a discrete time system. In this model, the plant represents the discrete-time components of the system, while the controller represents the discrete-event portions. The distinguishing feature of the plant is that it has a continuous state space, where the states take on values that are real numbers, and that the state evolves with time according to a set of difference equations

$$
\mathbf{x}(k+1)=F(\mathbf{x}(k), \mathbf{r}(k))
$$

where $\mathbf{x}(k) \in \Re^{n}$ and $\mathbf{r}(k) \in \Re^{m}$ are the state and input vectors respectively. $F: \Re^{n} \times \Re^{m} \rightarrow \Re^{n}$ is a continuous function.

The controller is a discrete event system which is modeled as a deterministic automaton $\{\tilde{S}, \tilde{X}, \tilde{R}, \delta, \phi\}$, as in Section 2.

The generator is defined by a set of smooth functionals, $h_{i}: \Re^{n} \rightarrow \Re$, and a function, $\alpha: \Re^{n} \rightarrow \tilde{X}$. Notice that unlike the continuous-time case, there is only one $\alpha$. Since the plant is discrete-time, the generator will not be able to identify the exact moment that a hypersurface is crossed. Rather, it identifies the first sample after a crossing has occurred. So the sequence of plant symbol instants is given by the following equations.

$$
\begin{aligned}
\tau_{e}[0] & =0 \\
\tau_{e}[n] & =\min \left\{k>\tau_{e}[n-1]: \exists i, h_{i}(\mathbf{x}(k)) \cdot h_{i}\left(\mathbf{x}\left(\tau_{e}[n-1]\right)\right)<0\right\}
\end{aligned}
$$

At each plant symbol instant a plant symbol is generated according to $\alpha$.

$$
\tilde{x}[n]=\alpha\left(\mathbf{x}\left(\tau_{e}[n]\right)\right)
$$

Not all plant events have to be represented by a plant symbol. Such events are silent, accompanied by the null symbol, $\epsilon$.

The actuator, $\gamma: \tilde{R} \rightarrow \Re^{m}$, converts a sequence of controller symbols to a plant input as follows.

$$
\mathbf{r}(k)=\sum_{n=0}^{\infty} \gamma(\tilde{r}[n]) I\left(k, \tau_{c}[n], \tau_{c}[n+1]\right)
$$


where $I(\cdot)$ is the following indicator function

$$
I\left(k, \tau_{c}[n], \tau_{c}[n+1]\right)=\left\{\begin{array}{ccc}
1 & \text { if } & \tau_{c}[n] \leq k<\tau_{c}[n+1] \\
0 & \text { if } & \text { otherwise }
\end{array}\right.
$$

and $\tau_{c}[n]$ is the sequence of control instants. Unlike the continuous-time case, here $\tau_{c}[n]$ is an integer. This sequence is based on the sequence of event instants, defined in equation 66 , according to

$$
\tau_{c}[n]=\tau_{e}[n]+\tau_{d}
$$

where $\tau_{d}$ is the delay associated with the controller.

\section{A.2 Digital Control in a Hybrid Framework}

In a digital control system, a continuous-time plant is controlled by a digital computer. The plant output is sampled and quantized to provide the input to the controller, and the control signal is passed through a zero-order hold to provide the plant input. Here, we show that such a digital control system is a special case of a hybrid control system. The more general framework of hybrid control systems is used to discuss problems encountered in digital control such as chattering and limit cycles. Section 3 presented a method for designing the interface of a hybrid control system using the natural invariants of the system. Here this method is used as an alternative to the usual quantization technique of digital control. An example illustrates the application of this method to a digital control problem; it also shows the application of a DES controller design approach to digital control.

\section{A.2.1 Modeling Digital Control}

A digital control system is a special case of a hybrid control system in which the plant, interface, and controller obey certain constraints. In a digital control system the events are triggered at regular (or, if desired, irregular) intervals of time. This gives rise to sampling and requires that there must be a clock present in the system. In digital control this clock is normally not modeled explicitly, but in a hybrid control framework it appears as part of the plant. A convenient way to do this is to use a two state oscillator such as,

$$
\dot{\mathbf{x}}_{c}=\left[\begin{array}{cc}
0 & \omega \\
-\omega & 0
\end{array}\right] \mathbf{x}_{c}
$$

where $\omega$ is the clock frequency. If required, $\omega$ can be made a function of the plant input, $\mathbf{r}(t)$, and then the clock frequency can be changed by the controller. To implement the sampling, hypersurfaces of the type

$$
h_{i}\left(\mathbf{x}_{c}\right)=k_{1} x_{c 1}+k_{2} x_{c 2}
$$

are used, where $k_{1}$ and $k_{2}$ are real constants. A single hypersurface with $k_{1}=1$ and $k_{2}=0$ would model the typical case where the sampling rate is given by $\omega / 2 \pi$. By adding more hypersurfaces, each with its own measurement function, $\alpha_{i}$, multirate sampling can be modeled.

When modeling digital control, the measurement function(s), $\alpha_{i}$, is a quantizer. The value of each state at the sampling instant is truncated or rounded and restricted to the range of values acceptable to the digital controller. For example, a controller implemented on an 8 bit computer might only have the capability to measure a state to 256 possible values. In that case we might have a set of plant symbols given by

$$
\tilde{X}=\{0,1, \ldots, 255\},
$$

and a measurement function given by

$$
\alpha_{1}(\mathbf{x}(t))=\operatorname{trunc}(\mathbf{x}(t)+128) \text { modulo } 256 .
$$

Quantization of this type will form a grid-like partition of the state space into regions, each associated with the same plant symbol. 
On the other side of the interface, the actuator models a zero-order hold. Controller symbols, representing quantized plant input values, are converted to piecewise constant plant inputs. In an example akin to the one given above, the set of controller symbols is given by

$$
\tilde{R}=\{0,1, \ldots, 255\}
$$

and $\gamma$ is given by

$$
\mathbf{r}\left(\tau_{c}[n]\right)=\tilde{r}[n]-128 .
$$

Finally, the controller is an automaton which implements the desired control strategy for the digital controller.

When a digital control system is modeled as a hybrid control system, the resulting hybrid control system has the following characteristics. First, the event triggering hypersurfaces are based only on the clock states. This means that they are "parallel" to all the other state axes of the plant. Because the state axes are parallel to the event triggering hypersurfaces, these states can vary arbitrarily without triggering an event and the sampling will occur at rates which are not dependent on the state of the plant (except for the state of the clock). The second characteristic of digital control is that the state measurements (plant symbols) are formed by quantizing the state values in a grid-like fashion. That is, each state is quantized individually to create a grid over the state space. Each cell in the grid is an n-dimensional box in the state space. These two characteristics actually result from the same phenomenon, except that they relate to different states in the plant. The first one applies to the state(s) of the clock and is a consequence of the clock based sampling. The second one applies to the remaining plant states as a result of the way states are quantized in the analog to digital converters found in digital control. Together the two characteristics create a grid style partition over the entire state space (including clock states) of the plant.

The designer is free to choose the sampling rate(s) and the quantization level(s) for each state. These choices determine the dimensions of the n-dimensional boxes which fill up the state space. What the designer cannot do is change the basic shape of the boxes which form the partition; they will always be rectangular. The limitations of digital control as compared to general hybrid control, that is, the requirement that the partition be a grid-like partition, give the advantage of a framework for design. The interface can be designed by selecting quantization levels and sampling rates for each state. Also, continuous-time control laws can be adapted for use in digital controllers via techniques like Euler's approximation or zero-order hold equivalence. The trade-off is that most hybrid control design possibilities cannot be realized in digital control because of the need for a grid-like partition.

\section{A.2.2 Problems Encountered with a Digital Controller}

The combination of sampling and quantized state measurements creates problems in digital control. Important problems are instability, chattering, and limit cycles. These effects can be described from the point of view of hybrid control systems.

- Instability - a digital control system can become unstable if the sampling interval is too long and/or the quantization levels are too far apart. In this case, an inappropriate control law was applied or an initially appropriate law became inappropriate over the course of one sampling interval. An inappropriate control law, in this case, is one which does not stabilize the system. For example, a particular control law may stabilize the system when it is in certain states but not in others. From the hybrid point of view we have a partition which is simply too large, either in the direction of time (long sampling interval) or in another direction (widely separated quantization levels). Not every plant state in the region will react the same way to a given control. This causes nondeterministic behavior. Thus a control law which is adequate for one part of the region may lead to instability when applied to a state in another part of the same region.

- Chattering - digital control systems, like hybrid systems in general can suffer from chattering. Chattering occurs along a surface which separates two different quantization levels or regions in the plant state space. If each of the two regions contains a control law which sends the plant state back to the 


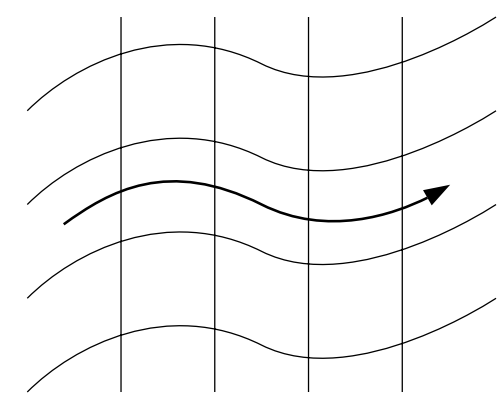

Figure 12: Nongrid-like Partition

other region, then the state will oscillate back and forth at the sampling rate. Again the culprit is non-deterministic behavior arising from state space partitioning. In this case, a pair of control laws which are probably appropriate for the states at the center of a region are not entirely appropriate for the states lying toward the boundaries of the region. The system with chattering may still behave appropriately in the large, though more control effort is expended. This is because even as the system chatters, it can also move along the boundary and eventually get off it.

- Limit cycles - a limit cycle occurs when the plant state can not reach the equilibrium point or when the equilibrium point is not associated with the appropriate control law to make it an invariant. The state oscillates about the equilibrium point.

\section{A.2.3 Multirate Sampling}

In multirate sampling, more than one clock cycle is allowed. Each clock cycle will have a sampling period and a state measurement function. This allows some states to be sampled at different rates from the others. In the state space of the hybrid control system, where time is included, multirate sampling will give rise to a partition with alternating layers of grids. As the system evolves, the state will lie in the layer associated with the current clock cycle and the grid associated with that layer will depend upon the measurement function (i.e. quantization) which goes with that clock cycle. Multirate sampling makes digital control more flexible, but it does not change the fact that there is always a grid-like partition of the state space. More on multirate sampling appears in the example.

\section{A.2.4 Towards solving the Problems of Digital Controller}

The central problem of digital control is the application of inappropriate controls; inappropriate meaning the control does not achieve the control goal. This is a result of the fact that the same control must be associated with all the states in a given region of the partition. There are two possible remedies. A different control policy can be chosen for a problem region of the partition. Or, the size of the partition regions can be adjusted so that the control policy is appropriate for the entire region. The size of the regions is determined by the sampling interval(s) and state measurement functions (quantization levels).

In digital control, the problem of choosing the appropriate control policy is many times handled by approximating an existing continuous control law. Since state space trajectories are not generally straight lines, they will not flow "neatly" through the grid-like partition of the digital control system. Trajectories in a given region of the partition will inevitably leave that region through more than one of the regions boundaries. This gives rise to non-deterministic behavior in the DES plant. The problem cannot be solved by changing the size of the region because it will still have the same grid-like shape. In hybrid control, the strategy is to shape the regions according to the system trajectories so as to control which boundaries the trajectories can pass through. This is not possible with a digital control system. Figure 12 shows an example of the added flexibility afforded by hybrid control over standard digital control.

The effect of playing with the sampling rate and quantization levels is to change the rate and amplitude of 
such effects as chattering and limit cycles. In general, faster sampling rates, coupled with finer quantization, will decrease the effects of these problems but there is no point at which they are eliminated or their nature qualitatively changes.

\section{A.2.5 Example: Double Integrator}

Since the problems encountered in digital control are largely a result of the quantization, it is reasonable to try to solve them by changing the way the quantization is done. The usual quantization forms a grid-like partition of the state space, forming an array of $n$-dimensional boxes each with its own symbol. The problem is that these quantization levels have no relationship to the state trajectories which flow through them. To reduce this problem, the grid-like partition can be replaced with a partition based on the natural invariants of the system. The following example explores the use of invariants for quantization.

In this example we have a double integrator which we wish to stabilize. The interface is designed to quantize based on system invariants and the controller is again designed with methods from hybrid control.

We start with a double integrator plant.

$$
\dot{\mathbf{x}}=\left[\begin{array}{ll}
0 & 1 \\
0 & 0
\end{array}\right] \mathbf{x}+\left[\begin{array}{l}
0 \\
1
\end{array}\right] \mathbf{r}
$$

The natural invariants of the system are be used in the quantization. Three possible inputs are used to control the double integrator, $\{-2,0,2\}$, and they are used to compute three invariant functions for the system. The invariant function associated with the input -2 is computed as follows. The control policy is

$$
\begin{aligned}
& f_{1}(\mathbf{x})=x_{2} \\
& f_{2}(\mathbf{x})=-2
\end{aligned}
$$

This gives the characteristic equation

$$
\frac{d x_{1}}{x_{2}}=\frac{d x_{2}}{-2}
$$

with a solution

$$
g_{1}(\mathbf{x})=x_{1}+.25 x_{2}^{2}
$$

The remaining two invariant functions can be computed similarly.

$$
\begin{aligned}
& g_{2}(\mathbf{x})=x_{2} \\
& g_{3}(\mathbf{x})=x_{1}-.25 x_{2}{ }^{2}
\end{aligned}
$$

The values of these invariant functions are quantized to form the plant symbols.

$$
\alpha(\mathbf{x})=.25 \operatorname{round}(4 g(\mathbf{x}))
$$

Now the quantization yields the partition shown in Figure 13 (i).

The controller for this case is similar to case 2 .

$$
\tilde{r}[k]=\left\{\begin{array}{ccc}
-2.0 & \text { if } & g_{3}(\mathbf{x})<0, g_{2}(\mathbf{x})<0 \\
2.0 & \text { if } & g_{2}(\mathbf{x})>0, g_{3}(\mathbf{x})>0 \\
0.0 & \text { otherwise } &
\end{array}\right.
$$

Figure 13 (ii) shows a state trajectory for this system. Notice that the chattering has been avoided and the controller easily follows from the interface design. 


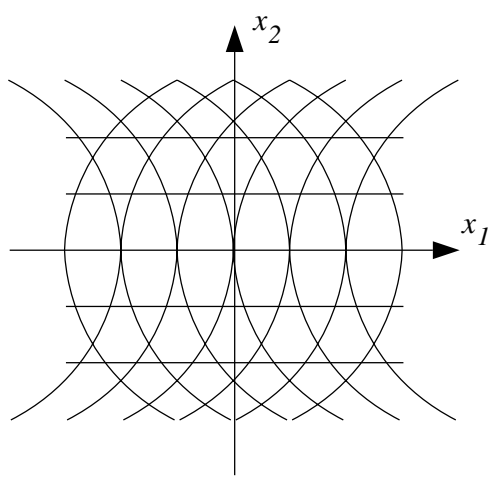

(i)

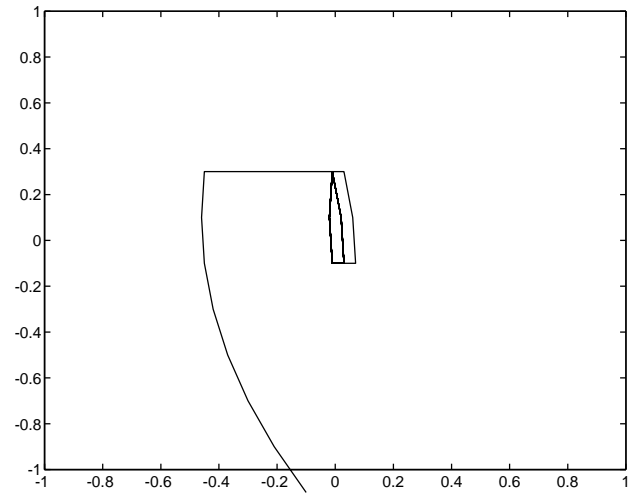

(ii)

Figure 13: (i) Quantization, (ii) State Trajectory

\section{References}

[1] R. Alur, C. Courcoubetis, N. Halbwachs, T. Henzinger, P.-H. Ho, X. Nicollin, A. Oliveiro, J. Sifakis, and S. Yovine. The algorithmic analysis of hybrid systems. Theoretical and Computer Science, 138:3-34, 1995.

[2] R. Alur, T. Henzinger, and E. Sontag, editors. Hybrid Systems III, Verification and Control, volume 1066 of Lecture Notes in Computer Science. Springer, 1996.

[3] P. Antsaklis, W. Kohn, M. Lemmon, A. Nerode, and S. Sastry, editors. Hybrid Systems V, volume 1567 of Lecture Notes in Computer Science. Springer, 1999.

[4] P. Antsaklis, W. Kohn, A. Nerode, and S. Sastry, editors. Hybrid Systems II, volume 999 of Lecture Notes in Computer Science. Springer, 1995.

[5] P. Antsaklis, W. Kohn, A. Nerode, and S. Sastry, editors. Hybrid Systems IV, volume 1273 of Lecture Notes in Computer Science. Springer, 1997.

[6] P. Antsaklis, X. Koutsoukos, and J. Zaytoon. On hybrid control of complex systems: A survey. European Journal of Automation, 32(9-10):1023-1045, 1998.

[7] P. Antsaklis and A. Nerode. Hybrid control systems: An introductory discussion to the special issue. IEEE Transanctions on Automatic Control, Special Issue on Hybrid Control Systems, 43(2):457-460, April 1998.

[8] P. Caines and Y.-J. Wei. Hierarchical hybrid control systems: A lattice formulation. IEEE Transactions on Automatic Control, 43(4):501-508, 1998.

[9] A. Chutinan and B. Krogh. Computing polyhedral approximations to flow pipes for dynamic systems. In Proceedings of the 37th IEEE Conference on Decision and Control, pages 2089-2094, Tampa, FL, Dec. 1998.

[10] J. Cury, B. Krogh, and T. Niinomi. Synthesis of supervisory controllers for hybrid systems based on approximating automata. IEEE Transactions on Automatic Control, 43(4):564-568, 1998.

[11] T. Henzinger. Hybrid automata with finite bisimulations. In Z. Fülöp and G. Gécgeg, editors, ICALP'95: Automata, Languages, and Programming. Springer-Verlag, 1995.

[12] T. Henzinger. The theory of hybrid automata. In Proceedings of the 11th Annual Symposium on Logic in Computer Science, pages 278-292. IEEE Computer Society Press, 1996. 
[13] J. E. Hopcroft and J. Ullman. Introduction to Automata Theory, Languages and Computation. AddisonWesley, 1979.

[14] A. Isidori. Nonlinear Control Systems. Springer-Verlag, 2nd edition, 1996.

[15] X. Koutsoukos, K. He, M. Lemmon, and P. Antsaklis. Timed Petri nets in hybrid systems: Stability and supervisory control. Journal of Discrete Event Dynamic Systems: Theory and Applications, 8(2):137$173,1998$.

[16] G. Lafferriere, G. Pappas, and S. Sastry. Hybrid systems with finite bisimulations. In P. Antsaklis, W. Kohn, M. Lemmon, A. Nerode, and S. Sastry, editors, Hybrid Systems V, volume 1567 of Lecture Notes in Computer Science, pages 186-203. Springer, 1999.

[17] J. Lunze, B. Nixdorf, and J. Schroder. Deterministic discrete-event representations of linear continuousvariable systems. Automatica, 35(3):396-406, 1999.

[18] R. Miller and A. Michel. Ordinary Differential Equations. Academic Press, New York, 1982.

[19] H. Nijmeijer and A. van der Schaft. Nonlinear Dynamical Control Systems. Springer-Verlag, 1990.

[20] A. Puri, V. Borkar, and P. Varaiya. $\epsilon$-approximation of differential inclusions. In R. Alur, T. A. Henzinger, and E. D. Sontag, editors, Hybrid Systems III, Verification and Control, volume 1066 of Lecture Notes in Computer Science, pages 362-376. Springer, 1996.

[21] J. Raisch and S. O'Young. Discrete approximation and supervisory control of continuous systems. IEEE Transactions on Automatic Control, 43(4):568-573, 1998.

[22] J. Stiver. Analysis and design of hybrid control systems. PhD thesis, Department of Electrical Engineering, University of Notre Dame, Notre Dame, IN, 1995.

[23] J. Stiver and P. Antsaklis. A novel discrete event system approach to modeling and analysis of hybrid control sysytems. In Proceedings of the 29th Annual Allerton Conference on Communication, Control and Computing, Univ. of Illinois at Urbana-Champaign, October 2-4 1991.

[24] J. Stiver, P. Antsaklis, and M. Lemmon. Hybrid control system design based on natural invariants. In Proceedings of the 34th IEEE Conference on Decision and Control, pages 1455-1460, New Orleans, LA, Dec. 1995.

[25] J. Stiver, P. Antsaklis, and M. Lemmon. Interface and controller design for hybrid control systems. In P. Antsaklis, W. Kohn, A. Nerode, and S. Sastry, editors, Hybrid Systems II, volume 999 of Lecture Notes in Computer Science, pages 462-492. Springer, 1995.

[26] J. Stiver, P. Antsaklis, and M. Lemmon. An invariant based approach to the design of hybrid control systems. In IFAC 13th Triennial World Congress, volume J, pages 467-472, San Francisco, CA, 1996.

[27] J. Stiver, P. Antsaklis, and M. Lemmon. A logical DES approach to the design of hybrid control systems. Mathl. Comput. Modelling, 23(11/12):55-76, 1996.

[28] M. Tittus and B. Egardt. Control design for integrator hybrid system. IEEE Transactions on Automatic Control, 43(4):491-500, 1998.

[29] F. Zhao. Extracting and representing qualitative behaviors of complex systems in phase spaces. Artificial Intelligence, 369(1-2):51-92, 1994. 